\title{
LOCALIZED RESIST HEATING DUE TO ELECTRON- BEAM PATTERNING DURING PHOTOMASK FABRICATION
}

\author{
by \\ ALEXANDER C. WEI \\ A thesis submitted in partial fulfillment of \\ the requirements for the degree of \\ MASTER OF SCIENCE \\ (MECHNICAL ENGINEERING) \\ at the \\ UNIVERSITY OF WISCONSIN-MADISON
}

2001 


\begin{abstract}
As the semiconductor industry continues to shrink the size microelectronic components, sources of critical dimension error that were unimportant in the past have surfaced, and must be resolved. Among these errors is the proximity heating effect. As an optical mask is patterned using an e-beam, there is heat diffusion away from the area being patterned. Due to the increase in temperature, the resist surrounding the patterned area increases in sensitivity and becomes more prone to development from scattered electrons. The unexpected development of resist and distortions due to thermal gradients can cause the final pattern to differ from the intended pattern.
\end{abstract}

Unfortunately, there is no method to predict the magnitude of these errors. Guess and check methods are not feasible in the production environment due to the limited number of chip manufacturing tools, and the need to produce saleable products on these tools. Consequently, a method is needed to predict the magnitude and location of these errors. The topic of this thesis is to investigate the thermal response of the optical mask due to direct patterning using a finite element program, ANSYS. The results from this thesis, resist temperature as a function of position and time, can then be combined with experimental data relating the temperature history of the resist with its sensitivity, and Monte Carlo simulations that predict the scattering of electrons as they penetrate an optical substrate to yield the percentage of resist development at every point on the mask. The results of this analysis can then be 
compared with the desired pattern. Any regions containing unacceptable errors can then be redesigned.

\section{Alexander C. Wei}

Under the supervision of Professors W. Beckman, J. Mitchell, and R. Engelstad. Department of Mechanical Engineering University of Wisconsin-Madison September 28, 2001 


\section{Acknowledgement}

The research in this thesis could not have been possible without the support provided by international SEMATECH, the Semiconductor Research Corporation, DARPA / ARL, and the Unversity of Wisconsin-Madison Graduate School.

To my advisors and supervisors, Professors William A. Beckman, John Mitchell, and Roxann L. Engelstad, I express my appreciation for their time and effort. Without their guidance and steering, I would have never come this far in my academic endeavors.

During the long days and nights I spent at the office, there were few human interactions other than with my fellow students in the Solar Energy lab and the Computational Mechanics lab. I thank these people for their generous assistance in all aspects of my studies and research, as well as for the human companionship.

Finally, thanks to my family who could always be counted on as a source of caring and support. 


\section{Table of Contents}

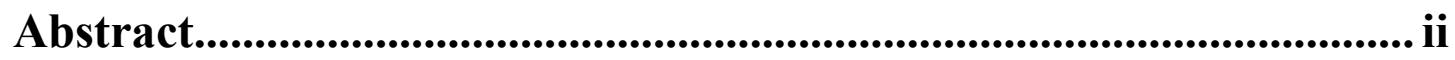

Acknowledgement....................................................................................... iv

Table of Contents ...................................................................................

List of Figures ...................................................................................... vii

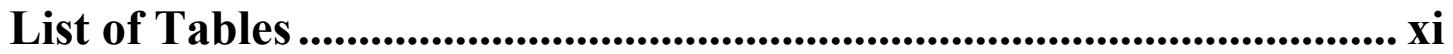

\section{Chapter 1}

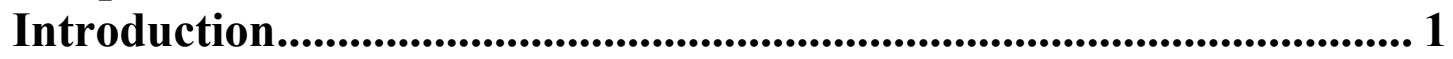

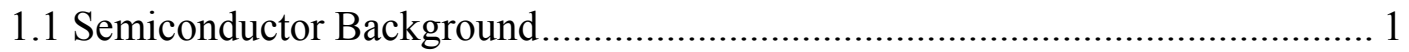

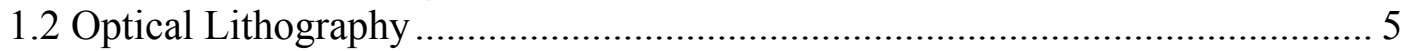

\section{Chapter 2}

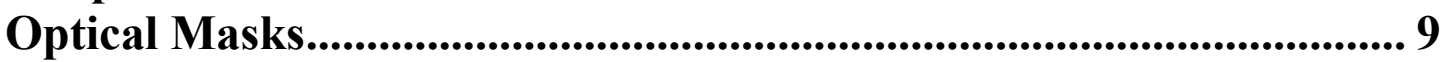

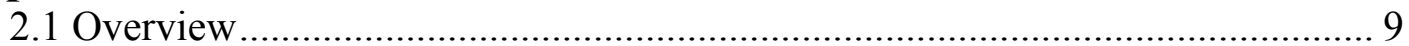

2.2 Mask Patterning Problems .......................................................................... 12

\section{Chapter 3}

Research Motivation and Model Development...................................... 18

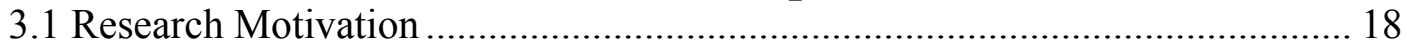

3.2 Model Development.............................................................................. 20

3.2.1 Physical Description of Model I ............................................................. 20

3.2.2 Pattern Description of Model I ............................................................. 21

3.2.3 Physical Description of Model II ........................................................... 22

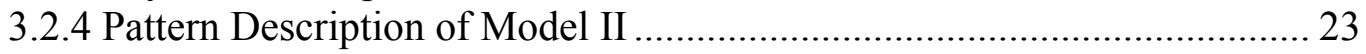

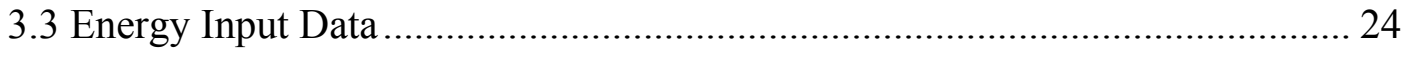

\section{Chapter 4}

Model I: Results and Analysis ..................................................................... 27

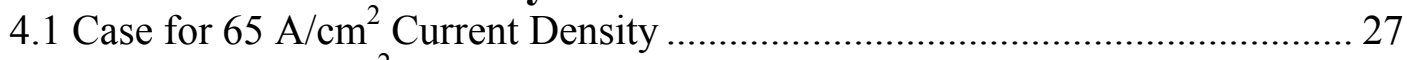

4.2 Case for $6.5 \mathrm{~A} / \mathrm{cm}^{2}$ Current Density ......................................................... 29 


\section{Chapter 5}

Model II: Results and Analysis

5.1 Visualization of Temperatures During Serpentine Writing....

5.2 Visualization of Temperatures During Unidirectional Writing ......................... 39

5.3 Temperature Histories at Various Points in the Patterned Subfield

\section{Chapter 6}

Summary and Future work ...............................................................55

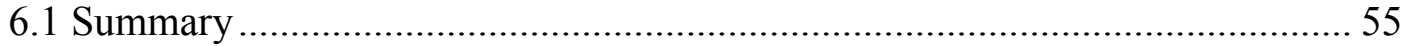

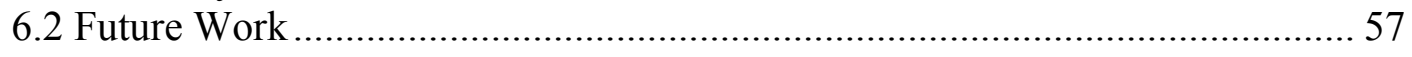

References............................................................................................................ 59 


\section{List of Figures}

Fig. 1.1 Single transistor schematic. ..................................................................... 2

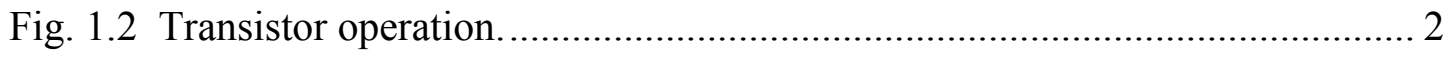

Fig. 1.3 Schematic of the wafer exposure process.............................................. 5

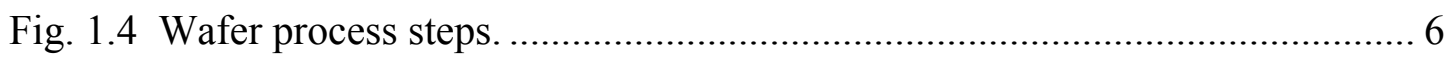

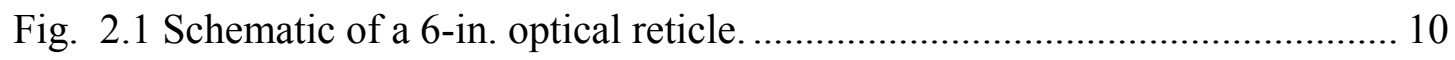

Fig. 2.2 Single feature patterning methodology ................................................... 11

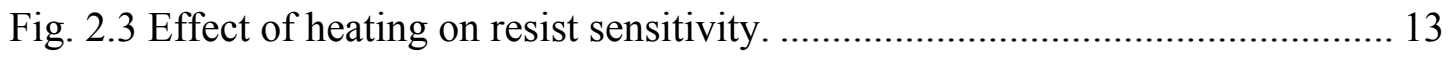

Fig. 2.4 Monte Carlo electron scattering simulation.............................................. 15

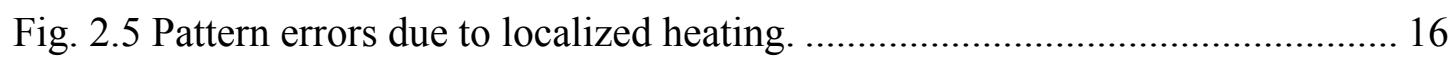

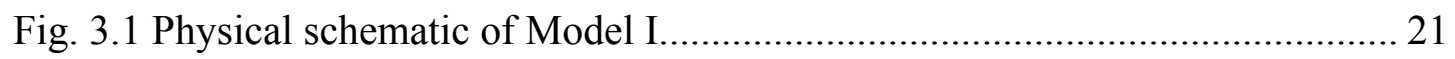

Fig. 3.2 Schematic of flash pattern for Model I ..................................................... 22

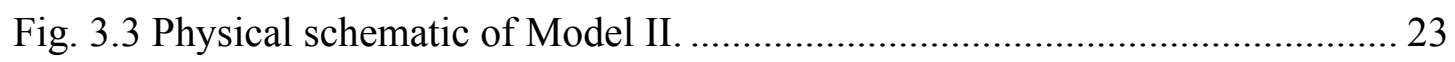

Fig. 3.4 Writing scheme and pattern schematic for Model II. .................................. 24

Fig. 3.5 Energy density for half-flash of Model II.............................................. 26

Fig. 4.1 Temperature contour plots on the top layer of the resist at the end of (a) 0.1 $\mu \mathrm{s}$, (b) $0.2 \mu \mathrm{s}$, and (c) $0.3 \mu \mathrm{s}$. Dose is $6.5 \mu \mathrm{C} / \mathrm{cm}^{2}$. Current density is $65 \mathrm{~A} / \mathrm{cm}^{2}$.

Fig. 4.2 Temperature contour plots of the plane through the cross section shown in Fig. 3.2 at the end of (a) $0.1 \mu \mathrm{s}$, (b) $0.2 \mu \mathrm{s}$, and (c) $0.3 \mu \mathrm{s}$. Dose is $6.5 \mu \mathrm{C} / \mathrm{cm}^{2}$. Current density is $65 \mathrm{~A} / \mathrm{cm}^{2}$.

Fig. 4.3 Temperature contour plots on the top layer of the resist at the end of (a) 1.0 $\mu \mathrm{s}$, (b) $2.0 \mu \mathrm{s}$, and (c) $3.0 \mu \mathrm{s}$. Dose is $6.5 \mu \mathrm{C} / \mathrm{cm}^{2}$. Current density is $6.5 \mathrm{~A} / \mathrm{cm}^{2}$. 30

Fig. 4.4 Temperature contour plots of the plane through the cross section shown in Fig. 3.2 at the end of (a) $1.0 \mu \mathrm{s}$, (b) $2.0 \mu \mathrm{s}$, and (c) $3.0 \mu \mathrm{s}$. Dose is $6.5 \mu \mathrm{C} / \mathrm{cm}^{2}$. Current density is $6.5 \mathrm{~A} / \mathrm{cm}^{2}$. 
Fig. 5.1 Temperature contour plots on the top layer of the resist for the $16^{\text {th }}$ flash of serpentine writing, $31 \mu$ s into the simulation. 35

Fig. 5.2 Temperature contour plots on the top layer of the resist for the $17^{\text {th }}$ flash of serpentine writing, $33 \mu$ s into the simulation.

Fig. 5.3 Temperature contour plots on the top layer of the resist for the $18^{\text {th }}$ flash of serpentine writing, $35 \mu$ s into the simulation.

Fig. 5.4 Temperature contour plots on the top layer of the resist for the $32^{\text {nd }}$ flash of serpentine writing, $63 \mu \mathrm{s}$ into the simulation.

Fig. 5.5 Temperature contour plots on the top layer of the resist for the $33^{\text {rd }}$ flash of serpentine writing, $65 \mu$ s into the simulation.

Fig. 5.6 Temperature contour plots on the top layer of the resist for the $34^{\text {th }}$ flash of serpentine writing, $67 \mu \mathrm{s}$ into the simulation.

Fig. 5.7 Temperature contour plots on the top layer of the resist for the $48^{\text {th }}$ flash of serpentine writing, $95 \mu$ s into the simulation.

Fig. 5.8 Temperature contour plots on the top layer of the resist for the $49^{\text {th }}$ flash of serpentine writing, $97 \mu \mathrm{s}$ into the simulation.

Fig. 5.9 Farfield view of temperature contour plots on the top layer of the resist for the $17^{\text {th }}$ flash of unidirectional writing, $33 \mu$ s into the simulation. 40

Fig. 5.10 Nearfield view of temperature contour plots on the top layer of the resist for the $17^{\text {th }}$ flash of unidirectional writing, $33 \mu$ s into the simulation.

Fig. 5.11 Farfield view of temperature contour plots on the top layer of the resist for the $49^{\text {th }}$ flash of unidirectional writing, $97 \mu$ s into the simulation.

Fig. 5.12 Temperature contour plots on the top layer of the resist for the $32^{\text {nd }}$ flash of unidirectional writing, $63 \mu$ s into the simulation. 42

Fig. 5.13 Temperature contour plots on the top layer of the resist for the $64^{\text {th }}$ flash of unidirectional writing, $127 \mu$ s into the simulation.

Fig. 5.14 Points at which temperature history plots for Figs. $5.15-5.30$ were generated. 
Fig. 5.15 Temperature history corresponding to Point 1 in Fig. 5.14 for serpentine writing. 45

Fig. 5.16 Temperature history corresponding to Point 1 in Fig. 5.14 for unidirectional writing. 45

Fig. 5.17 Temperature history corresponding to Point 2 in Fig. 5.14 for serpentine writing.

Fig. 5.18 Temperature history corresponding to Point 2 in Fig. 5.14 for unidirectional writing. 47

Fig. 5.19 Temperature history corresponding to Point 3 in Fig. 5.14 for serpentine writing. 48

Fig. 5.20 Temperature history corresponding to Point 3 in Fig. 5.14 for unidirectional writing. 48

Fig. 5.21 Temperature history corresponding to Point 4 in Fig. 5.14 for serpentine writing.

Fig. 5.22 Temperature history corresponding to Point 4 in Fig. 5.14 for unidirectional writing.

Fig. 5.23 Temperature history corresponding to Point 5 in Fig. 5.14 for serpentine writing. 50

Fig. 5.24 Temperature history corresponding to Point 5 in Fig. 5.14 for unidirectional writing. 50

Fig. 5.25 Temperature history corresponding to Point 6 in Fig. 5.14 for serpentine writing. 51

Fig. 5.26 Temperature history corresponding to Point 6 in Fig. 5.14 for unidirectional writing. 51

Fig. 5.27 Temperature history corresponding to Point 7 in Fig. 5.14 for serpentine writing.

Fig. 5.28 Temperature history corresponding to Point 7 in Fig. 5.14 for unidirectional writing. 
Fig. 5.29 Temperature history corresponding to Point 8 in Fig. 5.14 for serpentine

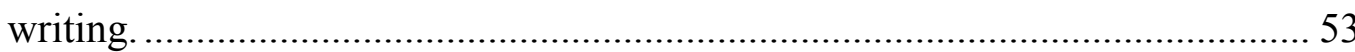

Fig. 5.30 Temperature history corresponding to Point 8 in Fig. 5.14 for unidirectional

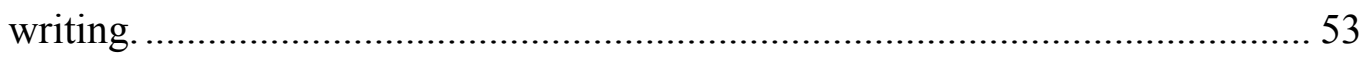




\section{List of Tables}

Table 3.1 Material properties of the individual layers utilized in Model I................ 21

Table 3.2 Material properties of the individual layers utilized in Model II................ 22 


\section{Chapter 1}

\section{Introduction}

\subsection{Semiconductor Background}

The integrated circuit has grown from a curiosity of electrical engineers to become one of the most important inventions that man has ever created. These circuits can be found in personal computers, cars, phones, satellites, and even children's toys. Almost every aspect of our lives is somehow influenced by the integrated circuit.

Behind these marvels of modern technology is the semiconductor. It is through the use of semiconductor materials that most of the technological advancements of the last half-century have been realized. A semiconductor is a material, which is neither a good conductor nor a good insulator of electricity. To change the electrical properties of a semiconductor, it is doped with another element. The type and level of doping determines whether the semiconductor is N-type (current is conducted by excess free electrons) or P-type (current is conducted by electron vacancies) ${ }^{1}$. Almost all microelectronics today are manufactured via the use of semiconductors.

One of the most important components created with semiconductors is the transistor, invented at Bell Laboratories in $1947^{2}$. A schematic of a single transistor is shown in Fig. 1.1. 


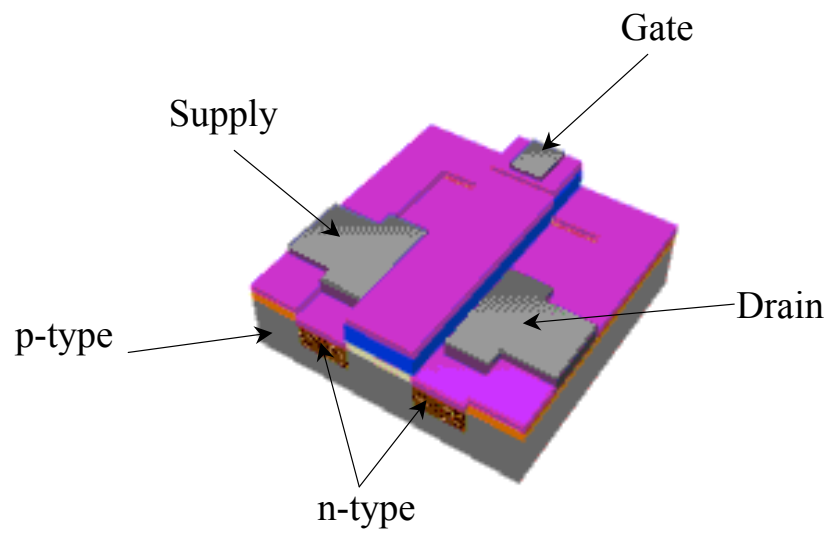

Fig. 1.1 Single transistor schematic ${ }^{3}$.
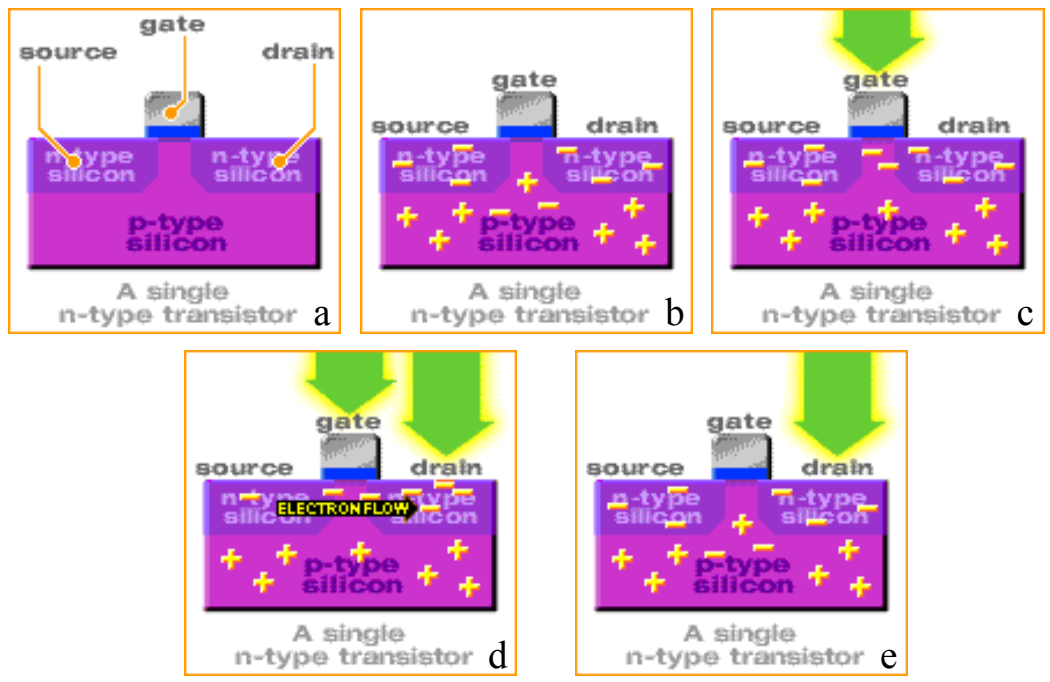

Fig. 1.2 Transistor operation ${ }^{4}$.

A transistor is essentially a switch. The basic operation is shown in Fig. 1.2. The transistor consists of two types of silicon. The n-type has an excess of electrons, and the p-type is electron deficient. When a voltage is applied to the gate, seen in Fig. 1.2c, the few electrons that remain in the p-type silicon are attracted to the positive charge and migrate towards the gate. This allows a current to flow from the 
source to drain, shown in Fig. 1.2d. This current flow can then be interpreted as a " 1 ". If the gate voltage is removed, as seen in Fig. 1.2e, current is no longer able to flow to the drain because the electrons from the p-type silicon migrate away from the gate, and are no longer able to act as the bridge for the current. The transistor output is then read as "0". The ability of the gate voltage to control the output to two different states allows it to act as a switch. These two states form the basis for the binary computations used by computers.

A transistor was initially designed as a single discrete component that could be placed into a circuit, and connected via wires to the other circuitry. However, each of the processors in modern personal computers contains tens of millions of these transistors along with associated connecting circuitry. Connecting individual transistors together to form these processors would not only be an immense task, but would create a large power hungry monstrosity. So, in 1959, scientists at Texas Instruments created the first integrated circuit ${ }^{5}$. An integrated circuit is most easily described as a collection of transistors that are manufactured directly from a single piece of material, and connected internally, without external wiring. The integrated circuit allowed the transistors to be smaller, faster, more reliable, and more energy efficient.

The microelectronics industry continuously tries to reduce the size of the transistors and circuits. The reason for this is twofold. First, increasing yield increases profits, so anything a manufacturer can do to increase yield is important. By reducing the size of the circuits, the overall chip size can be decreased. If the chip 
size is decreased, more chips can be manufactured onto a single wafer. Not only does this increase the gross yield per wafer, but it also decreases the percentage of chips lost to defects. To help visualize the idea of increasing yield by decreasing chip size, an example is given: Because manufacturing is not perfect and defects are bound to occur, there are an expected fixed number of defects per square unit of area. So, as an example, assume that 10 defects are expected on a standard 8-in. wafer, there are 10 chips (maximum) which will be defective. If the chips on the wafer are large, and only 20 chips can be manufactured on a single wafer, there is the possibility that $50 \%$ of the product is rejected. On the other hand, if the chips are small, and 100 chips can be printed on a wafer, the maximum percentage of product loss is $10 \%{ }^{6}$.

The second reason for miniaturization is for performance. The size reduction shortens the distance that electrons must travel in a transistor, decreasing the switching time. The faster a transistor can switch, the faster the overall processor can run. Reducing circuit dimensions also lowers the amount of heat generated by the circuit (because the elements are smaller and need less current to effect changes). Overheating of the primary processor in a computer can cause all forms of errors and failures to occur. Errors can materialize as unexpected application outputs, memory allocation problems, or disk problems. Examples of failures would be system crashes, lockups, or random rebooting. By reducing the energy requirement of the processor, and therefore reducing the heat output, the chip can be made to run faster without failure. 


\subsection{Optical Lithography}

If integrated circuits had to be made individually, the labor and costs required would be prohibitive for mass public consumption. Only select research institutions or large corporations would be able to afford microchips. To make chips affordable, there must be an efficient procedure to manufacture the chips quickly and in large quantities. To accomplish this feat, a technique called optical lithography is used. The figures below present the basic manufacturing processes used to produce an integrated circuit. A simple schematic of the exposure process is shown in Fig. 1.3, and the steps to processing the wafer are shown in Fig. 1.4.

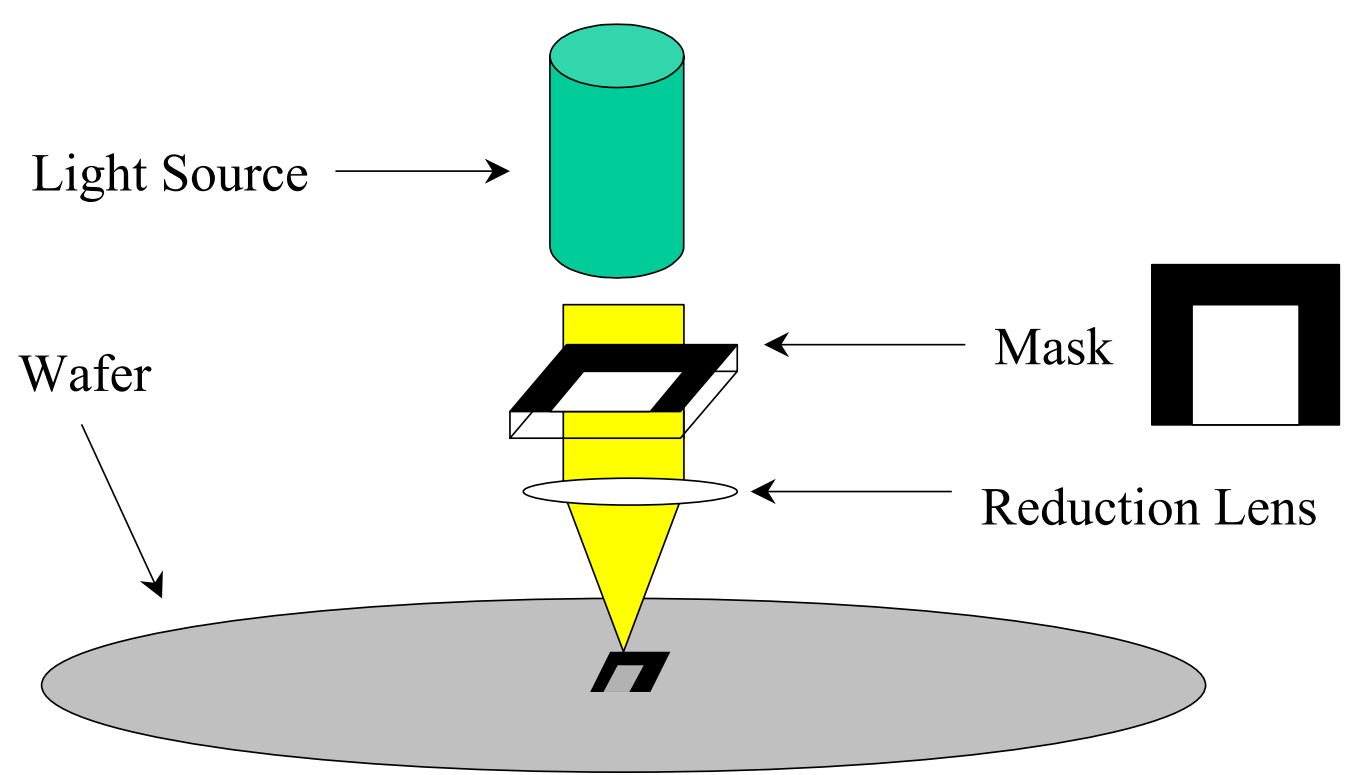

Fig. 1.3 Schematic of the wafer exposure process. 


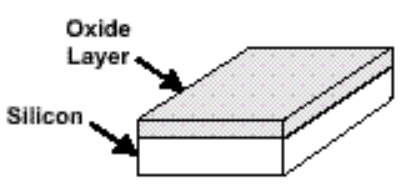

Silicon with

Oxide Layer

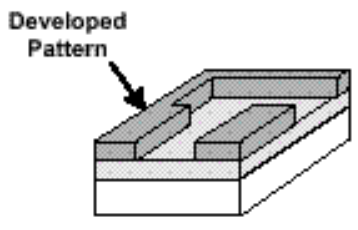

3) Develop Photoresist

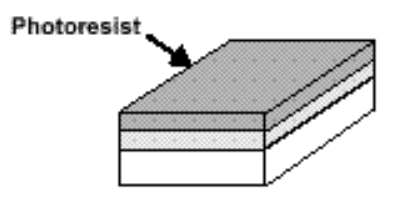

1) Coat with Photoresist

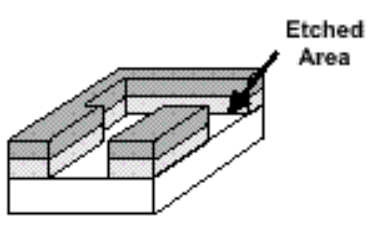

4) Etch Oxide Layer

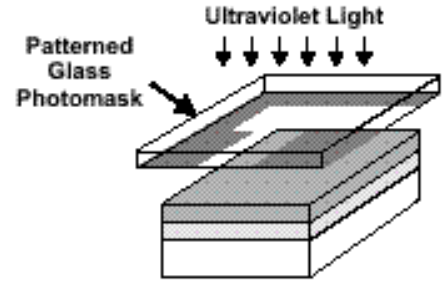

2) Expose Photoresist

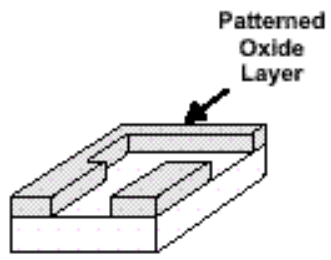

5) Strip Photoresist

Fig. 1.4 Wafer process steps ${ }^{7}$.

To print a circuit on a wafer of silicon, a beam of ultra-violet light is directed through a mask, as shown in Fig. 1.3. The pattern that is to be transferred to the wafer has been previously placed on the mask.

In optical lithography, masks were previously manufactured as "binary", meaning that the patterned portions of the mask prohibit light to pass through the mask, while the unpatterned portions allow the light through unchanged. As the feature size decreased, the industry developed phase-shifting masks, which have special patterned portions that change the phase of the light passing through to create destructive interference of the light waves. To keep explanations simple, only the binary mask will be described here. 
After the light passes through the mask, it carries with it the shadow of the pattern to be delivered to the wafer. The idea is much like the familiar shadow puppets created by placing your hands in front of a lamp, and projecting the image on a wall. This shadow is then directed through a reduction lens, usually $4 \times$ for optical lithography, which reduces the pattern and focuses the shadow onto the wafer. The reduction lens allows the pattern on the mask to be $4 \times$ larger than the required final pattern delivered to the mask. The $4 \times$ reduction is used because it is easier to produce larger patterns than smaller ones, therefore simplifying the complexity of the manufacturing process.

On the wafer is a layer of material called photoresist. Photoresist is a chemical that reacts to light. When the light that passed through the mask hits this photoresist layer on the wafer, it changes the resist's chemical structure. The exposed resist is capable of being etched away by a developing solution, while the unexposed areas are relatively unaffected.

After this exposure process, the wafer is put through various other steps, as illustrated in Fig. 1.4, to obtain the final desired features. After the exposed resist is developed away, another solution is used to etch away any exposed silicon dioxide. The remaining resist is then etched with another solution and the final feature is realized.

An alternate type of photoresist merits mention at this point. It is the negative resist. The resist mentioned thus far has been a positive resist, meaning that when it 
is exposed to light its chemical structure changes such that it is soluble in the developing solution. Negative resist, on the other hand, reacts in the opposite manner. When exposed to light, the negative resist becomes resistant to the effects of the developing solution. The result of this, then, is that only the unexposed areas of negative resist are removed during the development phase.

Many repetitions of the process of applying resist, exposing, and etching are used to create a layered three-dimensional structure that makes up an integrated circuit. On each wafer, many chips can be manufactured. To create multiple chips, the wafer is stepped to different locations and exposed again. After all the chips are completed, the wafer is cut into pieces, each individual chip is tested, packaged, and then sent out to the consumers. 


\section{Chapter 2}

\section{Optical Masks}

\subsection{Overview}

The mask briefly described in Chapter 1 is arguably one of the more important components used in optical lithography. It is responsible for carrying the pattern of the features that are to be produced on the finished wafer. Without a mask, the only way to print a feature would be to directly pattern the wafer using electron beams or other direct write tools. This direct write method has been unattractive in the past due to the slow speed at which direct-write produces features as compared with mask exposure.

The strength of using a mask lies in the fact that a pattern only needs to be exposed once on the mask using a direct write strategy. After writing the mask, the entire pattern can be transferred to the wafer at one time by the methods illustrated in Fig. 1.3.

In Fig. 2.1, a schematic of a mask with approximate dimensions is shown. The mask is fabricated starting from fused silica glass 0.25 in. thick. A layer of chrome $80 \mathrm{~nm}$ thick is then deposited on the glass. The next material to be deposited is a layer of photoresist $400 \mathrm{~nm}$ thick. These deposited layers and the glass substrate comprise what is known as the mask blank. The next step in the mask fabrication process is the patterning. 


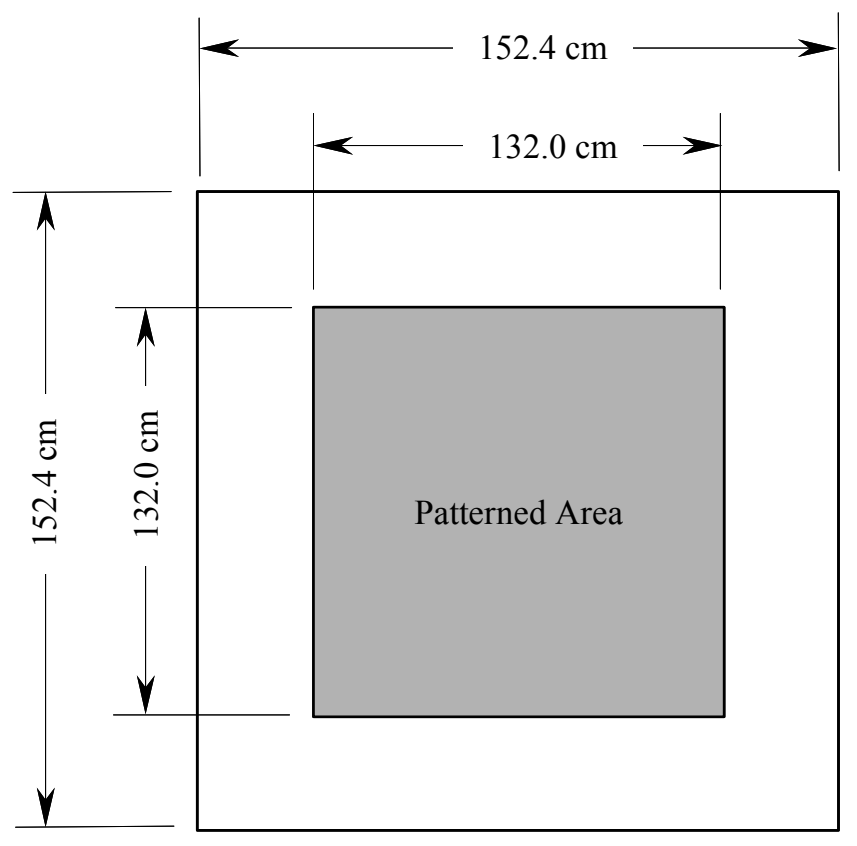

Fig. 2.1 Schematic of a 6 -in. optical reticle.

The patterning methodology of a small feature is shown in Fig. 2.2. The numbered areas represent the four flashes of an electron beam that are required to write the desired pattern, which in this case is an inverted U. This is an example of a direct write strategy. Note that to create this $U$ pattern on the mask requires 4 flashes of the e-beam, but to transfer the pattern to the wafer only requires one exposure from the mask to the wafer. Now if we consider using a direct write method on the wafer, it would also require 4 flashes to create this $U$ shape, but because one mask is used multiple times to create multiple chips, the time savings from using a mask is immense. 


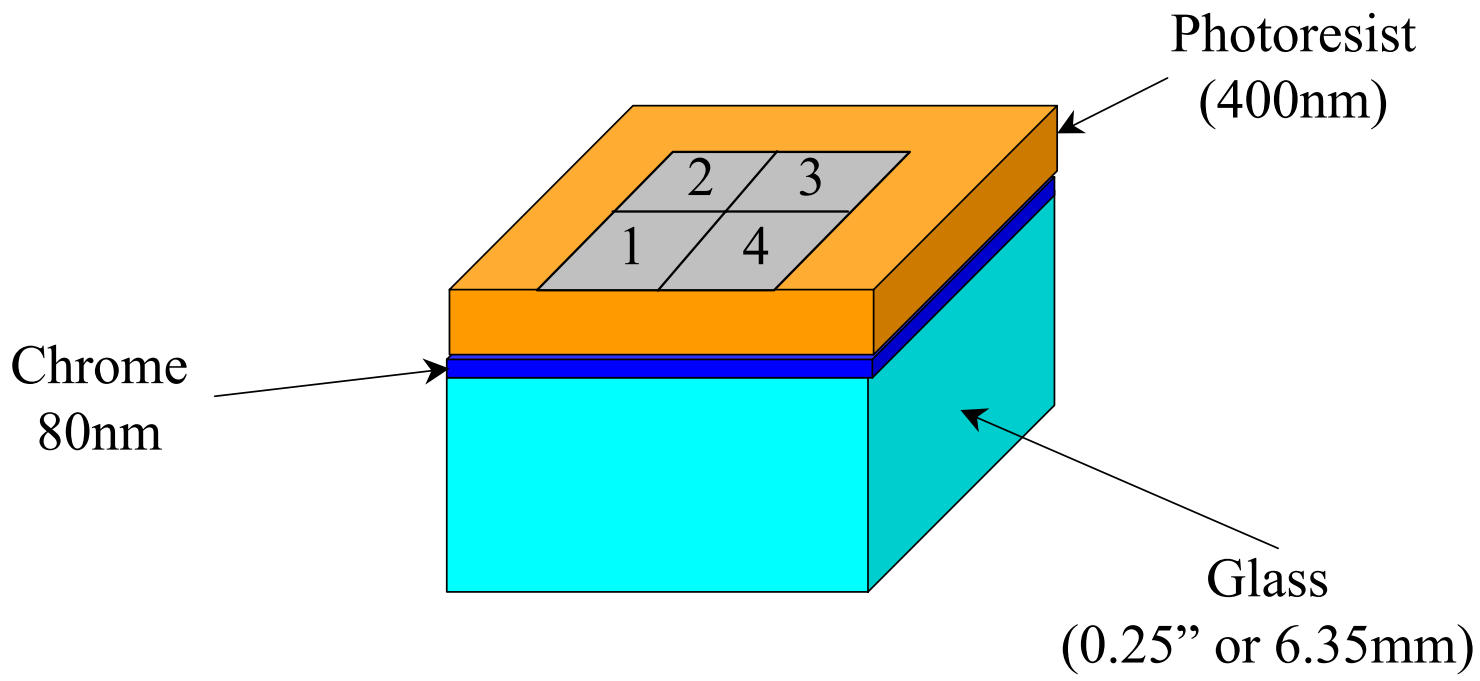

Fig. 2.2 Single feature patterning methodology.

For the case concerning positive resists, when the electron beam (e-beam) is flashed onto the mask, development occurs. An e-beam develops resist by using its high energy electrons to break the chemical bonds in the resist, causing chemical changes that allow it to be etched away by the developing solution. Resists have a threshold value that determines if they are developed or not. This is referred to as its sensitivity. An increase in sensitivity means that a resist requires a lower number of electrons to hit it before it becomes soluble to the developing chemicals.

Once the exposed photoresist is etched away, portions of the underlying chrome are exposed. Another chemical is used to etch away the exposed chrome, and then the remaining resist is removed. In the end, the only material left is a thin chrome layer in the shape of a U pattern on the glass. 


\subsection{Mask Patterning Problems}

It was discussed in Chapter 1 why microchip designers want to reduce the size of the manufactured chips. Throughout the last decade, the manufacturers have been able to meet the stricter manufacturing requirements that are needed to produce these features effectively. A crossroads is soon to be reached however. In the not too distant future, the current form of lithography will have encountered a physical wall that will prevent its further use as a tool for microchip fabrication.

Very briefly, the problem is that the light used to expose the mask and transfer the pattern to the wafer will have a wavelength greater than that of the dimensions of the feature to be written. A comparable feat would be to try and draw a thin line using a large marker.

To be able to further miniaturize the circuits, an alternate production technique must be used. Currently, the industry is researching many ideas for a next generation lithography (NGL) technique, but until a one has been tested and proven, use of optical lithography must be extended.

To extend the life of optical lithography, all factors that contribute to critical dimension $(\mathrm{CD})$ error must be eliminated or minimized. $\mathrm{CD}$ error is basically the difference between what you want and what you get. For instance, if we are trying to write a $100 \mathrm{~nm}$ line and we get a $150 \mathrm{~nm}$ line, that is a $50 \mathrm{~nm}$ CD error. Among the factors that affect $\mathrm{CD}$ is the undesired development of e-beam resist due to the localized heating effect during the mask fabrication process ${ }^{8,9}$. 
Two factors are present that lead to heating as a problem. The first is the increased sensitivity of the resist as it gets heated ${ }^{10}$. In Fig. 2.3 below is the result of an experiment showing the effect of heating on resist sensitivities.

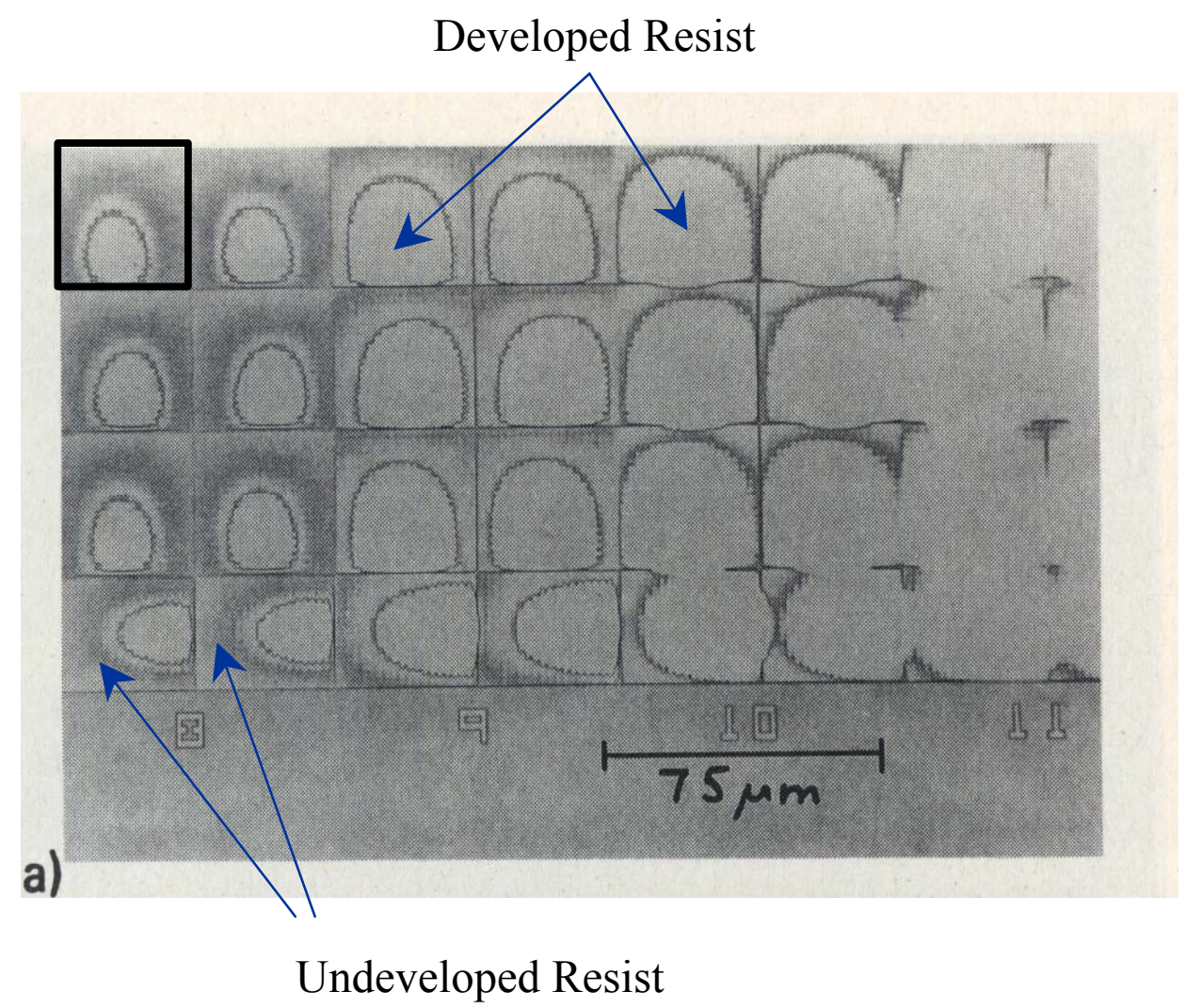

Fig. 2.3 Effect of heating on resist sensitivity ${ }^{11}$.

The experiment was performed by writing on a mask, but with a dose under the resist's development threshold. The writing began at the subfields on the left edge and continued rightward. A representative subfield is shown highlighted by the box in the figure. All subfields were written individually in what is called a serpentine strategy. For example, in the highlighted subfield, the beam began in the top left corner of the subfield and the zigzagged left to right, and then downwards until the 
entire subfield was patterned. The beam then moved to the next subfield, and began the serpentine pattern again. In the bottom row of subfields, the serpentine pattern zigzagged up and down, and then rightward. What would be expected then is that when the mask is developed, none of the resist will be etched away, and no chrome will be exposed. The results however show that there are large patches of exposed chrome. In fact, towards the end of the writing, there is almost no resist left.

An explanation is that when patterning the mask, the e-beam deposits energy to the resist and sublayers. This large localized energy infusion from the e-beam is needed to develop the resist, but at the same time appears as heat that causes a significant temperature rise around the currently exposed area. As the heat diffuses away from the high temperature regions, surrounding areas undergo a temperature rise $^{12,13}$. The heat diffuses throughout the reticle faster than the patterning e-beam. Therefore, before the e-beam actually arrives to pattern an area, that area of resist has already undergone a physical increased sensitivity change due to its temperature history. An increased sensitivity means that it now requires fewer electrons (lower dose) to develop the same resist. Due to this increased sensitivity in the surrounding areas, unexpected development of the resist may occur ${ }^{11,14,15}$.

Increased sensitivity of the resist would not normally be a problem, but because of electron scattering, patterning errors like the ones shown above can occur. Scattering is caused because the electrons hit molecules as they travel through the substrate. Every time an electron hits a molecule it bounces off in another direction. 
Shown in Fig. 2.4 is a simulation showing the paths of a few electrons as they hit a mask.

The primary incident beam spreads out as it makes its way through the substrate. This is called forward scattering. Some electrons, though, hit the molecules at strange angles and end up going upwards. This is backscattering.

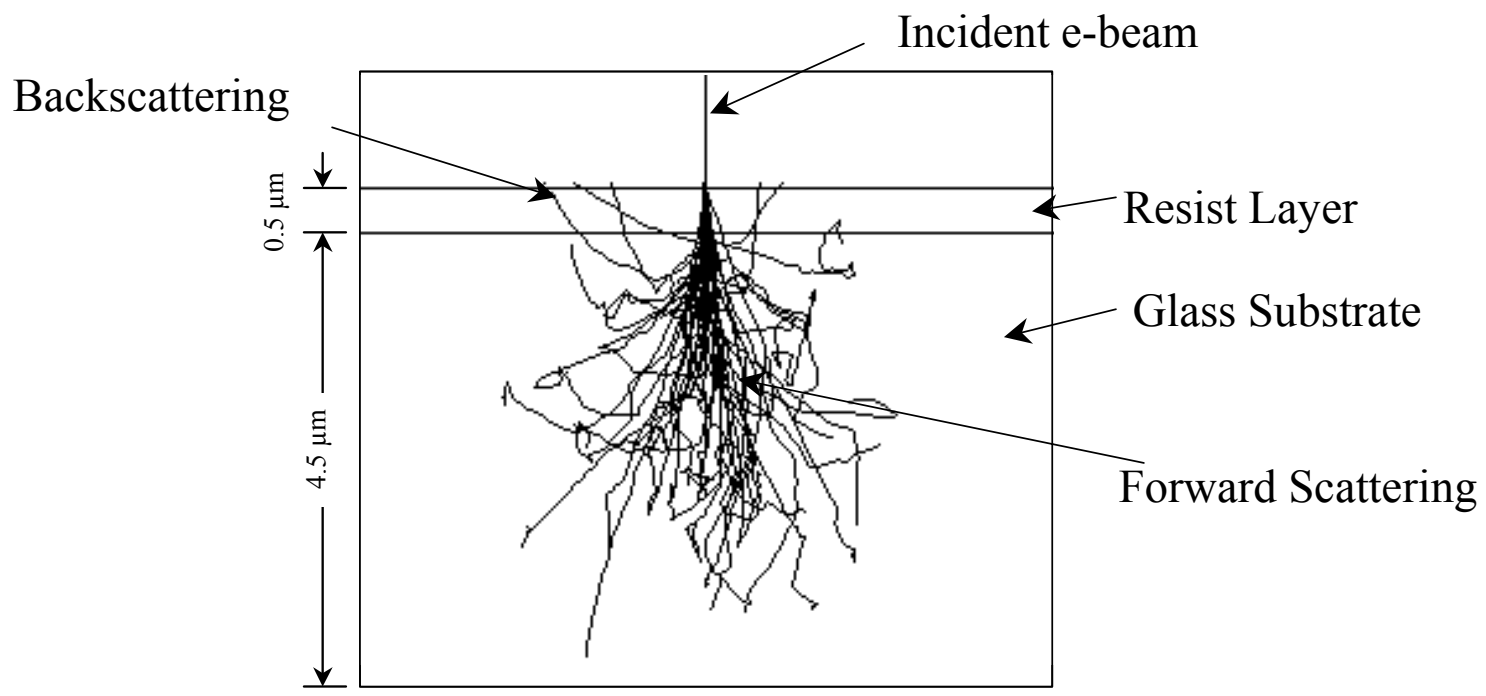

Fig. 2.4 Monte Carlo electron scattering simulation ${ }^{16}$.

Because the electrons spread, development outside the desired written area may occur. Again referring to Fig. 2.4, the desired written area is the size of the incident beam, which is fairly small. Most of the electrons will hit resist molecules in a small area surrounding this incident location and cause development. Some of these electrons, though, will scatter outside this immediate area. Usually these rogue electrons do not cause the resist to surpass the development threshold and nothing 
happens. However, if the sensitivity of the resist has been increased due to heating, these few electrons may cause the resist to develop.
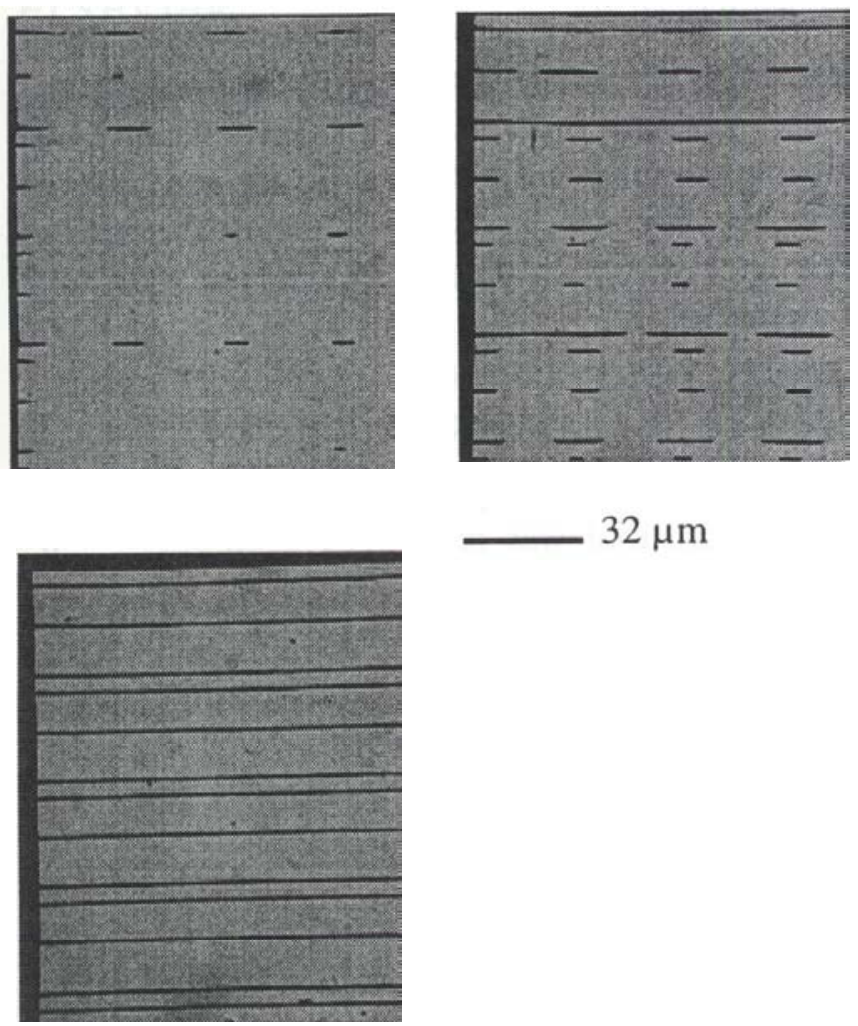

$32 \mu \mathrm{m}$

Fig. 2.5 Pattern errors due to localized heating ${ }^{17}$.

Prior research has indicated that these resist development problems do exist, and can have major implications for the future of e-beam pattering ${ }^{18,19,20}$. For example, in Fig. 2.5 above, the bottom picture represents the pattern that is desired. The black lines are $1 \mu \mathrm{m}$ wide lines of undeveloped resist. To create this pattern, the e-beam patterns subfields much like in Fig. 2.3, but it skips over the areas where the resist is to remain. 
Ideally, after the developed resist is etched away, only the $1 \mu \mathrm{m}$ lines remain. If, however, writing parameters are incorrectly chosen, results such as those shown in the top two figures may happen. What causes these line breaks is that the localized heating affects the sensitivity of the resist enough so that even though the e-beam never passes directly over the lines, the beam widening caused by forward scattering and backscattered electrons from the writing around the lines causes development. 


\section{Chapter 3}

\section{Research Motivation and Model Development}

\subsection{Research Motivation}

At the end of Chapter 2, evidence was given that localized heating may be an important factor in the CD error budget. The next step is to analyze the extent of the CD errors caused by localized heating and to determine techniques that can be used to minimize these errors. There are two paths one can follow to examine localized heating. One method is experimental and the other is through the use of computer simulations.

Many problems are encountered if the experimental path is chosen. To perform experiments, one must obviously have a machine or tool to perform the experiments. Unfortunately, the tools used by industry to pattern the masks cost many millions of dollars and are constantly being used to produce saleable products. Rarely is the tool ever stagnant. Any time used for experimental use detracts from the production of revenue generating products, which is undesirable in the short term. Also, because of the extremely fast time frames, on the order of $1 \mu$ s per flash, and small flash sizes, on the order of $2 \mu \mathrm{m}$, it becomes impossible to record accurate temperature data from the experiments. So, in the end, the only analysis possible with experiments is to compare the final feature shapes with the desired shapes and approximate the effect of localized heating. 
Simulations clearly are more accurate than experimental methods. Although simulations require computational time, the time is not nearly as valuable as the time required on the tools. Another major strength of simulations is the ability to visualize results. Temperature contours can not only be mapped at any point in time, but can also be seen through cutting planes. These flexible resources effectively give the user the power to see how the heat is diffusing at anytime during the simulation anywhere in the model; not just at the surface, as would be the case in experiments if temperatures could even be measured experimentally. Also of interest is that the temperature histories can be extracted for any location in the substrate. The temperature histories are of relevance because the sensitivity of a resist depends on its entire temperature history and not just the peak temperatures reached. Finally, another strength of simulations stems from the fact that any parameter in the simulation can be changed, and the effect of this change can be recorded. There would be no external costs associated with this test. Experimentally, it is very difficult if not impossible to change any conditions other than writing order and current density.

The research presented here investigates the capability of finite element (FE) methods to predict the heat transfer in various optical mask configurations due to ebeam patterning. Currently, there is a commercially available software package, TEMPTATION $^{21}$, that is capable of simulating temperature rises and predicting the equivalent doses received by the resist in optical masks ${ }^{17,22}$. The FE methods presented here provides an alternate means of predicting the temperature rises due to 
e-beam patterning. The strengths of the TEMPTATION ${ }^{21}$ program are its speed and ease of use. The FE method, on the other hand, provides users with unlimited control of the simulation parameters such as boundary conditions and mesh sizes, and also provides the ability to perform thermo-mechanical modeling analysis if desired.

\subsection{Model Development}

Finite element models have been developed on ANSYS ${ }^{23}$ and ABAQUS ${ }^{24}$ commercial finite element platforms. Various simulations were run to test the models. The ability of the FE models to resolve fine temperature details is discussed first. The second set of results demonstrates the ability of the FE models to run complete subfield patterning simulations. Descriptions of the models and the patterns exposed are provided in the following sections.

\subsubsection{Physical Description of Model I}

Two models were developed to test the FE method's ability to accurately predict the temperatures encountered during localized heating. The first model, shown below in Fig. 3.1, was designed to demonstrate the ability of FE models to simulate the response of relatively fine features. In particular, the model was constructed using elements as small as $200 \mathrm{~nm} \times 200 \mathrm{~nm} \times 40 \mathrm{~nm}$. The overall model dimensions were chosen to be large enough so that thermal effects were not observed at the adiabatic perimeters. Two avenues of energy dissipation are possible in the model; they are conduction within the solid model and radiation to the surroundings, 
held at $298 \mathrm{~K}$. The materials in order from top to bottom are: PMMA, chrome, and fused silica. Properties for the various materials are shown in Table 3.1.

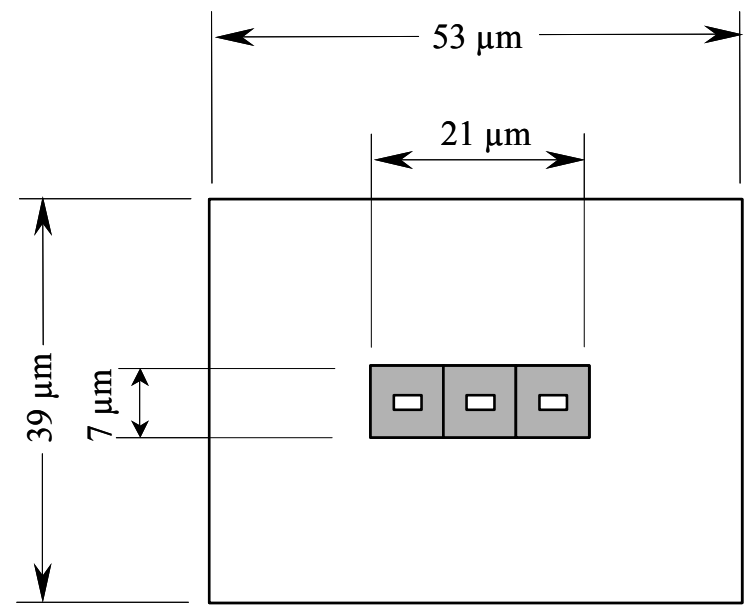

Fig. 3.1 Physical schematic of Model I.

Table 3.1 Material properties of the individual layers utilized in Model I.

\begin{tabular}{|c|c|c|c|c|c|}
\hline Material & $\begin{array}{c}\text { Thickness } \\
(\mu \mathrm{m})\end{array}$ & $\begin{array}{c}\text { Density } \\
\left(\mathrm{g} / \mathrm{cm}^{3}\right)\end{array}$ & $\begin{array}{c}\text { Thermal Conductivity } \\
(\mathrm{W} / \mathrm{cm}-\mathrm{K})\end{array}$ & $\begin{array}{c}\text { Specific Heat } \\
(\mathrm{J} / \mathrm{g}-\mathrm{K})\end{array}$ & Emissivity \\
\hline PMMA & 0.40 & 1.19 & 0.0021 & 1.460 & 0.84 \\
\hline Chrome & 0.08 & 7.19 & 0.6290 & 0.465 & \\
\hline $\mathrm{SiO}_{2}$ & 30.00 & 2.20 & 0.0140 & 0.750 & \\
\hline
\end{tabular}

\subsubsection{Pattern Description of Model I}

Three "Donut" shaped flashes, shown in Fig. 3.2, were applied to the model. Each flash consisted of a $7 \mu \mathrm{m} \times 7 \mu \mathrm{m}$ flashed area with a $3 \mu \mathrm{m} \times 1 \mu \mathrm{m}$ unflashed area in the center. The dosage to the resist was $6.5 \mu \mathrm{C} / \mathrm{cm}^{2}$ and was applied by a beam with an energy of $50 \mathrm{kV}$. Two cases with different beam current densities were run: $6.5 \mathrm{~A} / \mathrm{cm}^{2}$ and $65 \mathrm{~A} / \mathrm{cm}^{2}$. The e-beam shot time for each flash was calculated by dividing the dosage by the beam current densities. In the above two cases for 
example, the shot time for the $6.5 \mathrm{~A} / \mathrm{cm}^{2}$ is $1 \mu \mathrm{s}$, and the shot time for the $65 \mathrm{~A} / \mathrm{cm}^{2}$ case is $0.1 \mu \mathrm{s}$. No delay time between flashes was considered for this simulation.

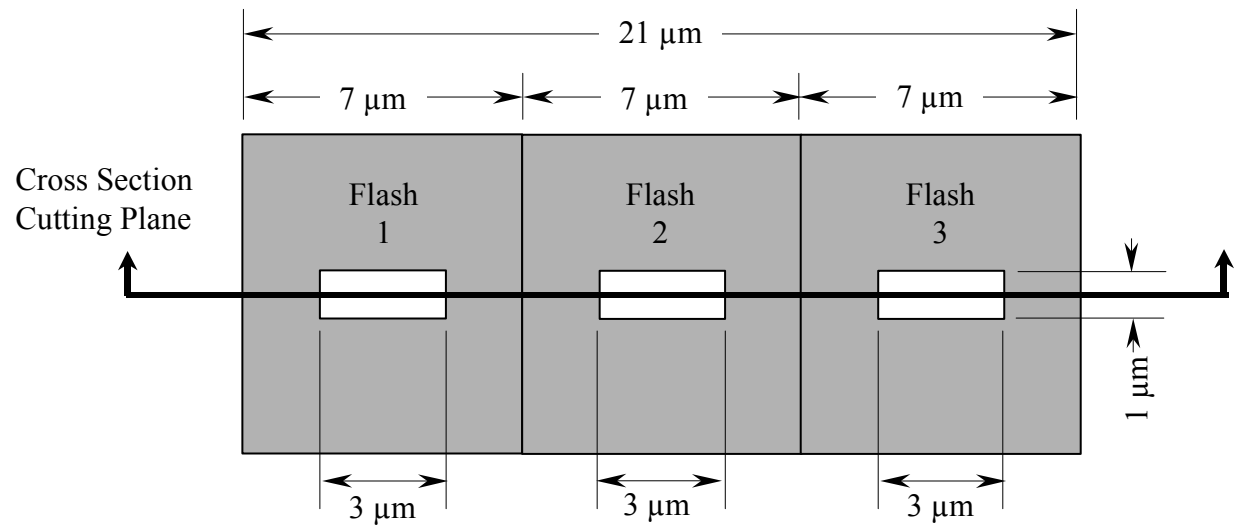

Fig. 3.2 Schematic of flash pattern for Model I

\subsubsection{Physical Description of Model II}

The second model, as shown in Fig. 3.3, was used to verify that the FE method was capable of simulating complete patterning of a subfield. For this simulation, the pattern is a checkerboard. The same model boundary conditions exist in Model II as in Model I. The materials in order from top to bottom are: ZEP7000, $\mathrm{CrO}_{2} \mathrm{~N}_{3}$, chrome, and fused silica. Properties for the various materials are shown in Table 3.2.

Table 3.2 Material properties of the individual layers utilized in Model II.

\begin{tabular}{|c|c|c|c|c|c|}
\hline Material & $\begin{array}{c}\text { Thickness } \\
(\mu \mathrm{m})\end{array}$ & $\begin{array}{c}\text { Density } \\
\left(\mathrm{g} / \mathrm{cm}^{3}\right)\end{array}$ & $\begin{array}{c}\text { Thermal } \\
\text { Conductivity } \\
(\mathrm{W} / \mathrm{cm}-\mathrm{K})\end{array}$ & $\begin{array}{c}\text { Specific Heat } \\
(\mathrm{J} / \mathrm{g}-\mathrm{K})\end{array}$ & Emissivity \\
\hline $\mathrm{ZEP} 7000$ & 0.45 & 1.10 & 0.0019 & 0.980 & 0.84 \\
\hline $\mathrm{CrO}_{2} \mathrm{~N}_{3}$ & 0.03 & 5.21 & 0.6290 & 0.465 & \\
\hline $\mathrm{Chrome}$ & 0.07 & 7.19 & 0.6290 & 0.465 & \\
\hline $\mathrm{SiO}_{2}$ & 50.00 & 2.20 & 0.0140 & 0.750 & \\
\hline
\end{tabular}




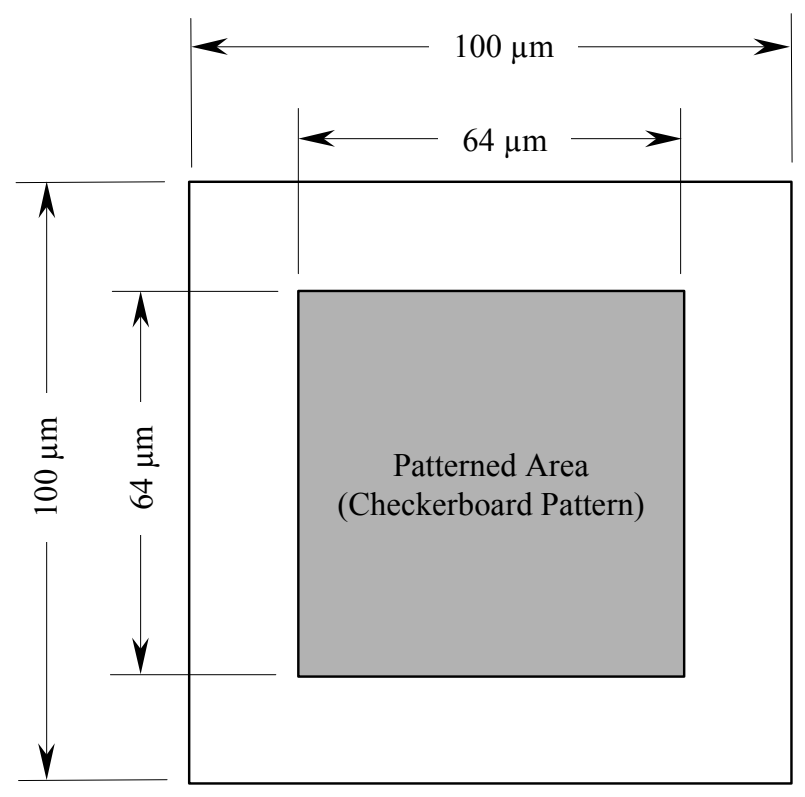

Fig. 3.3 Physical schematic of Model II.

\subsubsection{Pattern Description of Model II}

The pattern was written using a $2 \mu \mathrm{m} \times 2 \mu \mathrm{m}$ shot size. The location of the first flash was in the lower right bottom corner. Two simulations were run corresponding to two patterning schemes. A schematic for the first few rows of serpentine patterning is shown in Fig. 3.4. Also run was a unidirectional patterning in which the beam follows the same sequence as for the serpentine patterning for the first row. At the end of the first row, however, the beam returns to the beginning of the second row before it begins to pattern again. It continues to reset itself to the beginning of each row that is to be patterned. Both simulations consisted of a total of 64 shots. In reality, the entire pattern would consist of 512 shots. A long time would have been required for such a large simulation, and only one-eighth of the complete 
pattern could be simulated. The beam delay time between shots was $1 \mu$ s. The dosage to the resist was $9.6 \mu \mathrm{C} / \mathrm{cm}^{2}$ and was applied by a beam with an energy of $50 \mathrm{kV}$ and a current density of $9.6 \mu \mathrm{C} / \mathrm{cm}^{2}$, yielding a $1 \mu$ s flash time.

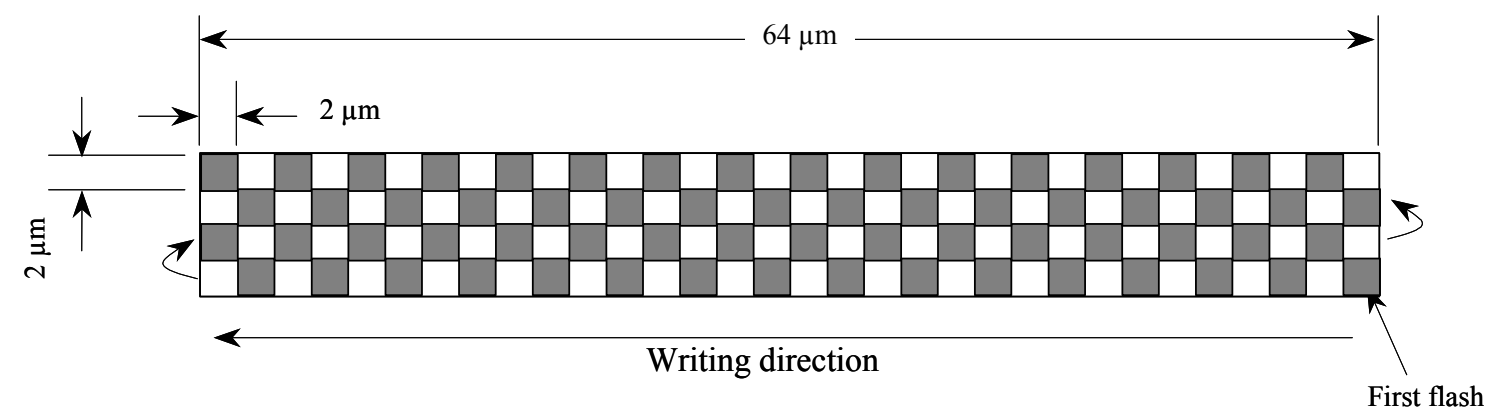

Fig. 3.4 Writing scheme and pattern schematic for Model II.

\subsection{Energy Input Data}

In both models, the energy input data used to simulate each flash were obtained through the use of proprietary programs developed at Intel Corp. A basic overview of how the simulation program operates follows.

First, the program simulates the paths of a large number of electrons as they interact with a material stack similar to the one used in the finite element simulations. All electrons enter the material stack at the same location. Monte Carlo methods are then used to predict the paths of each of these electrons as they make their way through the substrates. A plot of the results would look similar to Fig. 2.4.

From the electron paths, the program can predict the amount of energy that is left within each point in the volume due to electron-molecule interactions. The 
energy is then normalized to one electron to create a base from which the various flash geometries are constructed.

In order to create the $2 \mu \mathrm{m} \times 2 \mu \mathrm{m}$ square flash used in Model II, the number of electrons necessary to reach the development dose must be determined. In this example, the necessary dose is $9.6 \mu \mathrm{C} / \mathrm{cm}^{2}$. There are $6.24 \mathrm{e}+12$ electrons per $\mu \mathrm{C}$. This results in approximately $6 \mathrm{e}+13$ electrons $/ \mathrm{cm}^{2}$. Each of the elements from the normalized distribution is multiplied by this number to give the total energy deposited. This result, however, is only valid for a point equal to the projected area of an electron. The entire $2 \mu \mathrm{m} \times 2 \mu \mathrm{m}$ area is obtained by taking the total energy distribution and propagating it to fill the required flash area. All overlapping regions are then summed to get the final energy distribution. The program then breaks up the simulated field into small three-dimensional elements. For example, in Model II, the elements for the resist, chrome oxide, and chrome layers were $20 \mathrm{~nm} \times 20 \mathrm{~nm} \times 10$ $\mathrm{nm}$, and the elements for the fused silica layer were $20 \mathrm{~nm} \times 20 \mathrm{~nm} \times 100 \mathrm{~nm}$.

The raw data obtained from Intel for Model II came in four files, one for each material layer in the simulation. For each $2 \mu \mathrm{m} \times 2 \mu \mathrm{m}$ flash, the simulation region was $6 \mu \mathrm{m} \times 6 \mu \mathrm{m}$ in the $\mathrm{x}-\mathrm{y}$ direction and $450 \mathrm{~nm}, 30 \mathrm{~nm}, 70 \mathrm{~nm}$, and $50 \mu \mathrm{m}$ in depth for the ZEP7000, chrome oxide, chrome, and fused silica layers, respectively. The total number of data points for the four files was $49,950,000$ for a total of 538.43 megabytes of data. Every data point represents the value of energy deposited in each the above mentioned elements. 
Unfortunately, to run the multiflash simulations as required in Model II would require a number in excess of 6 billion elements. The computing power and resources need to solve a problem of this size does not currently exist today. To reduce the number of elements required an averaging of groups of adjoining elements. Programs were written in PERL script to accomplish this averaging.

A half-symmetry, cross-sectional view of the energy density for one flash of Model II is shown in Fig. 3.5 below.

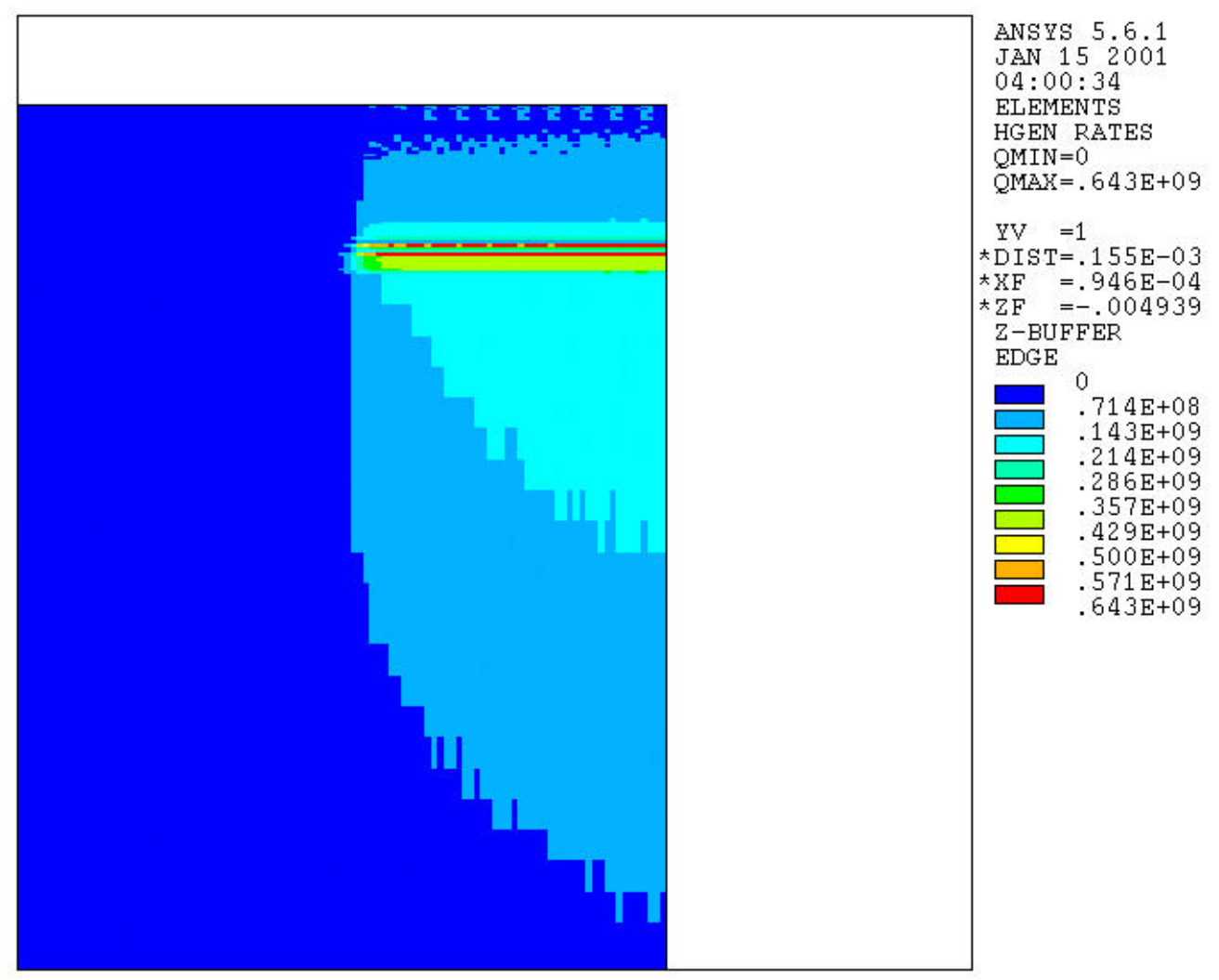

Fig. 3.5 Energy density for half-flash of Model II. 


\section{Chapter 4}

\section{Model I: Results and Analysis}

\subsection{Case for $65 \mathrm{~A} / \mathrm{cm}^{2}$ Current Density}

Simulation results for the case in which the current density was $65 \mathrm{~A} / \mathrm{cm}^{2}$ are shown in Figs. 4.1 and 4.2. Shown in Fig. $4.1 \mathrm{a}, \mathrm{b}$, and $\mathrm{c}$ are the temperatures at the top of the resist at the end of $0.1,0.2$, and $0.3 \mu$ s into the simulation, respectively. These times correspond to the end of each shot. Shown in Fig. 4.2 a, b, and c are cross-section views at the same time intervals of $0.1,0.2$, and $0.3 \mu$ s as in Fig. 4.1, but through the cutting plane shown in Fig. 3.2.

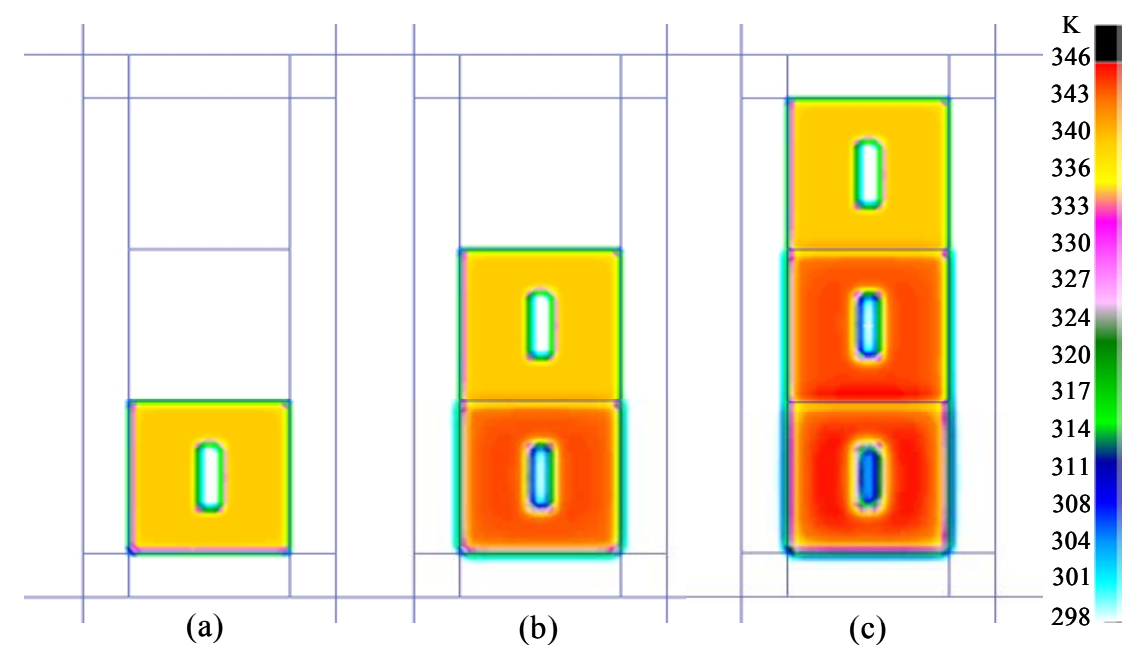

Fig. 4.1 Temperature contour plots on the top layer of the resist at the end of (a) 0.1 $\mu \mathrm{s}$, (b) $0.2 \mu \mathrm{s}$, and (c) $0.3 \mu \mathrm{s}$. Dose is $6.5 \mu \mathrm{C} / \mathrm{cm}^{2}$. Current density is $65 \mathrm{~A} / \mathrm{cm}^{2}$. 

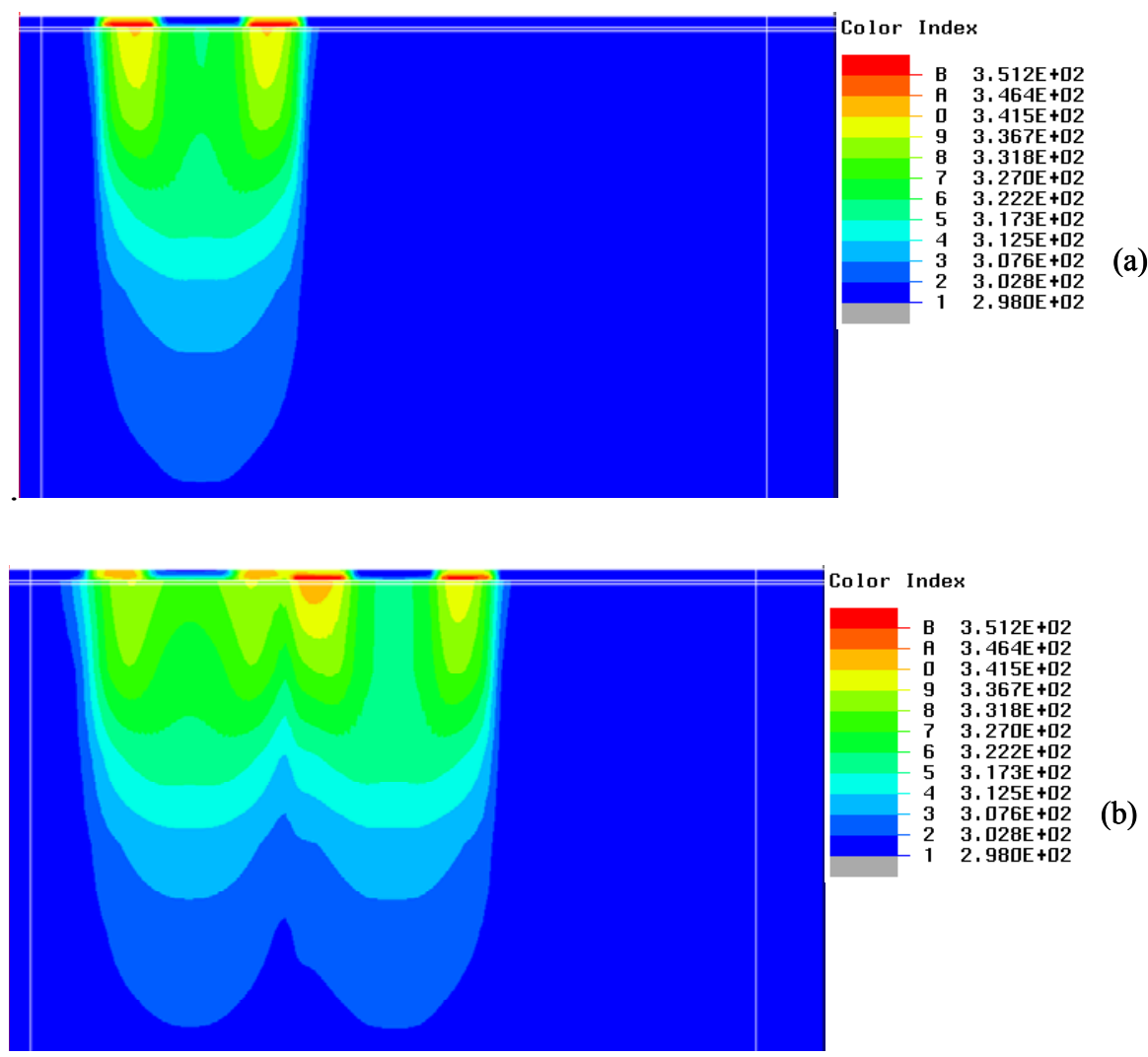

Color Index

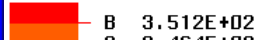

ค $3,464 \mathrm{E}+02$

A $3.464 \mathrm{E}+02$

d $3.415 \mathrm{E}+02$
$\mathbf{9} .367 \mathrm{E}+02$

$83.318 \mathrm{E}+02$

$\begin{array}{r}3.270 E+02 \\ \hline\end{array}$

$63.222 \mathrm{E}+02$

5 3. $173 \mathrm{E}+02$

4 3. $125 \mathrm{E}+02$

3 3. $776 E+02$ (b)

$\begin{array}{ll}2 & 3.028 E+02 \\ 1 & 2.980 E+02\end{array}$

(b)

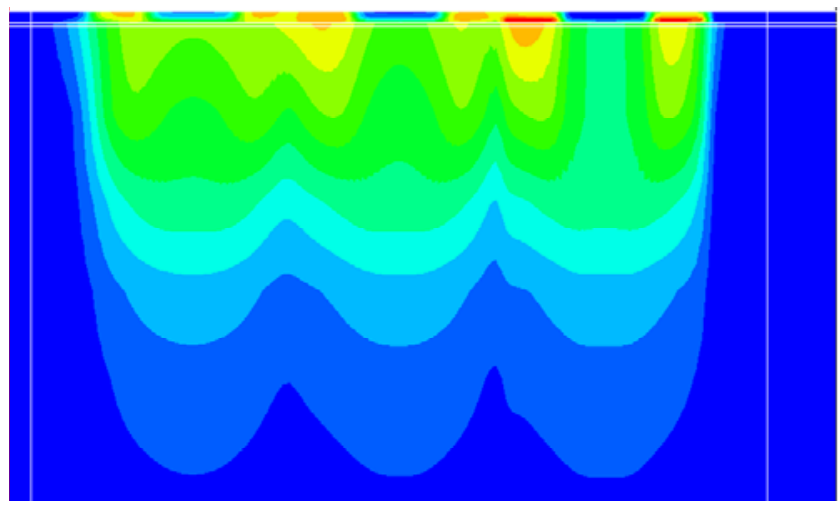

Color Index

- B $3.512 \mathrm{E}+02$

$3.464 \mathrm{E}+02$

3. $415 \mathrm{E}+02$

3. $367 \mathrm{E}+02$

3. $318 \mathrm{E}+02$

3. $270 \mathrm{E}+02$

3. $222 \mathrm{E}+02$

3. $173 \mathrm{E}+02$

$3.076 E+02$ (c)

3. $028 \mathrm{E}+02$

$2.980 E+02$

(a)

Fig. 4.2 Temperature contour plots of the plane through the cross section shown in Fig. 3.2 at the end of (a) $0.1 \mu \mathrm{s}$, (b) $0.2 \mu \mathrm{s}$, and (c) $0.3 \mu \mathrm{s}$. Dose is $6.5 \mu \mathrm{C} / \mathrm{cm}^{2}$. Current density is $65 \mathrm{~A} / \mathrm{cm}^{2}$. 
Initially the maximum temperature is not at the top of the resist. Both figures show that surface temperatures at the end of any given flash are $340 \mathrm{~K}$, while the maximum temperature is $356 \mathrm{~K}$ in the center of the resist layer. This is because the ebeam deposits most of the energy near the top of the chrome layer and the bottom of the resist layer due to the high density of the chrome. After $0.1 \mu \mathrm{s}$, the temperature at the top of the resist layer increases to $346 \mathrm{~K}$ due to the diffusion of heat.

\subsection{Case for 6.5 $\mathrm{A} / \mathrm{cm}^{2}$ Current Density}

Simulation results for the case in which the current density was $6.5 \mathrm{~A} / \mathrm{cm}^{2}$ are shown in Figs. 4.3 and 4.4. Shown in Fig. $4.3 \mathrm{a}, \mathrm{b}$, and $\mathrm{c}$ are the temperatures at the top of the resist at the end of $1.0,2.0$, and $3.0 \mu$ s into the simulation, respectively. These times correspond to the end of each shot. Shown in Fig. 4.4 a, b, and c are cross section views at the same time intervals of 1.0, 2.0, and $3.0 \mu$ s as in Fig. 4.3 but through the cutting plane shown in Fig. 3.2. The maximum temperature in this case is at the top of the resist because the longer writing time allows heat to dissipate further. The maximum temperatures range from $339 \mathrm{~K}$ to $349 \mathrm{~K}$ due to proximity heating effects. 


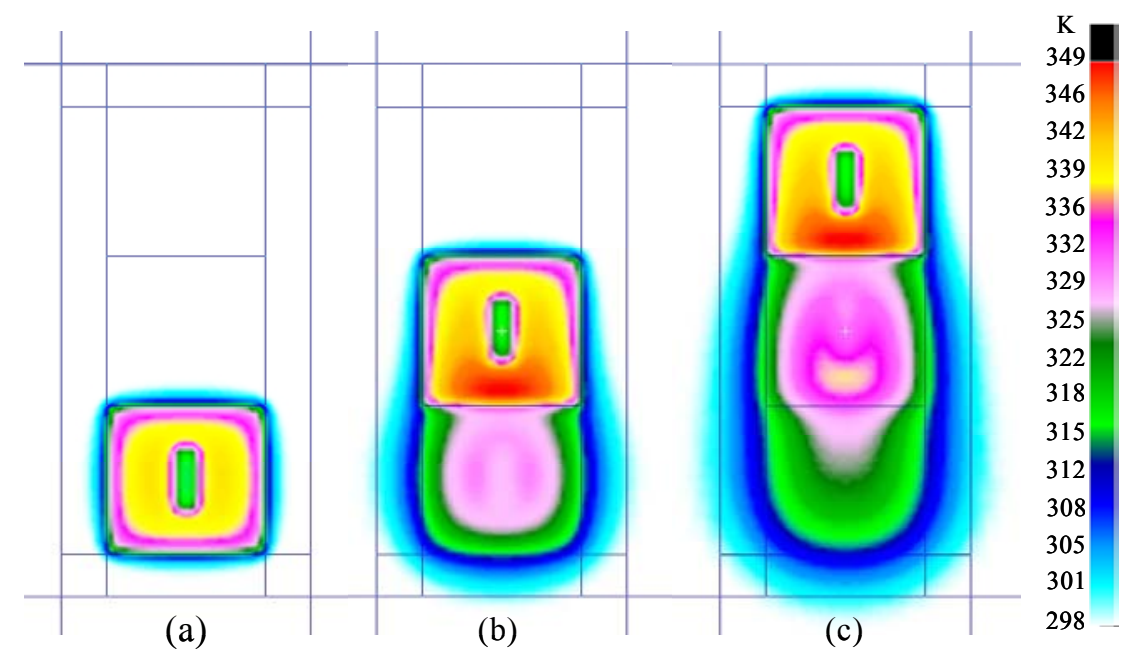

Fig. 4.3 Temperature contour plots on the top layer of the resist at the end of (a) 1.0 $\mu \mathrm{s}$, (b) $2.0 \mu \mathrm{s}$, and (c) $3.0 \mu \mathrm{s}$. Dose is $6.5 \mu \mathrm{C} / \mathrm{cm}^{2}$. Current density is $6.5 \mathrm{~A} / \mathrm{cm}^{2}$.

The high spatial resolution possible with the FE model, which is attributable to the small element sizes, allows for the observance of interesting features. In Figs. 4.2 - 4.4, the fine temperature contours in the resist layer can be resolved. In Figs. 4.1 - 4.3, it is possible to see blurring of the temperature contours at the edges of the flash and temperature contour corner rounding at the corners of the inner unflashed rectangle.

More importantly, the effect of one flash on the next consecutive flash can be seen in Figs. 4.1 - 4.4. It is not significant in the first simulation for a $65 \mathrm{~A} / \mathrm{cm}^{2}$ current density because the $0.1 \mu$ s flash time is too quick for any significant heat diffusion to occur. 

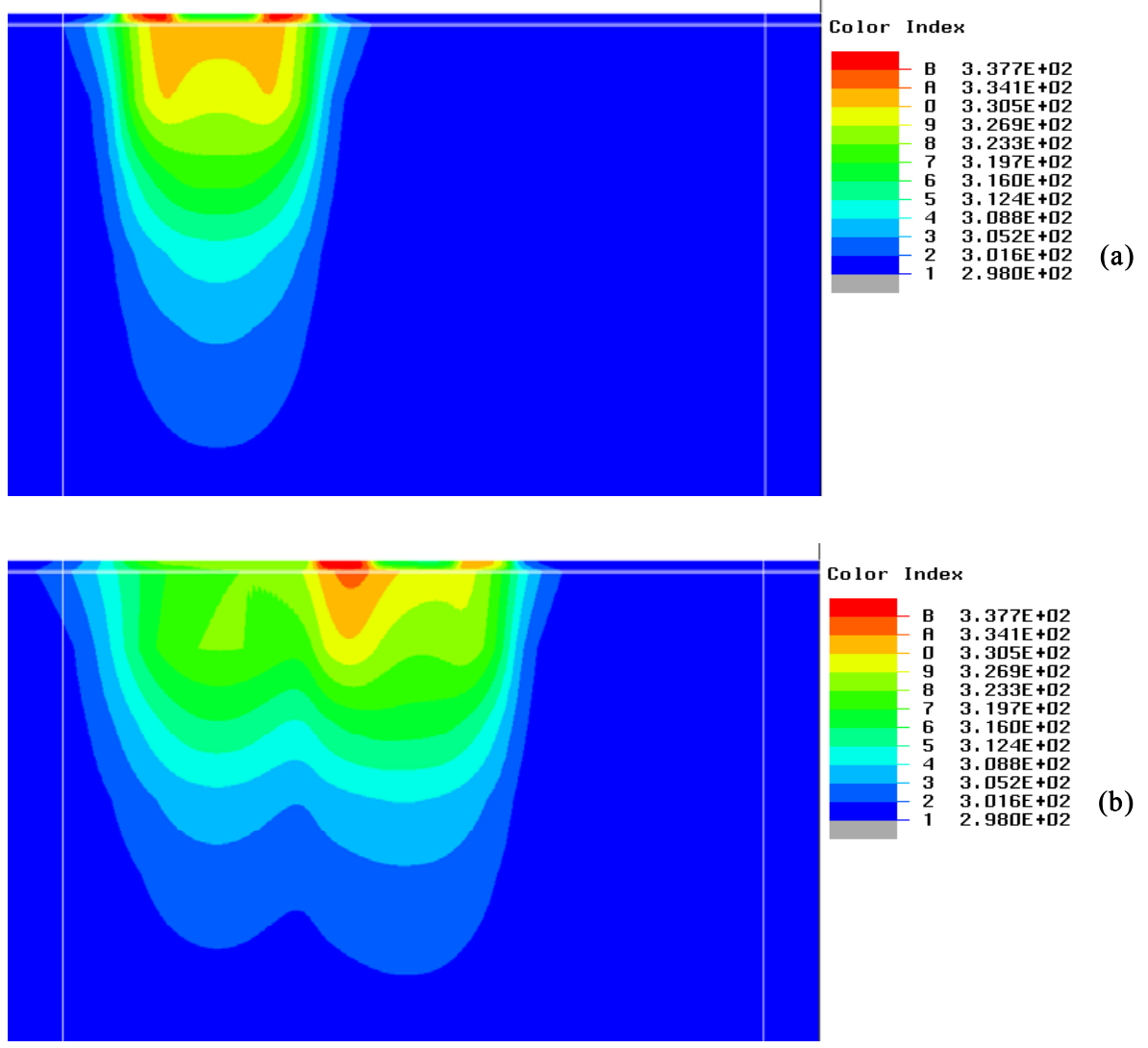

Color Index

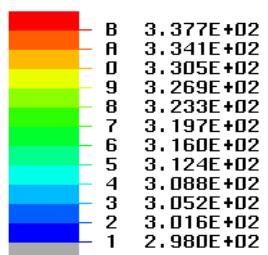

(b)

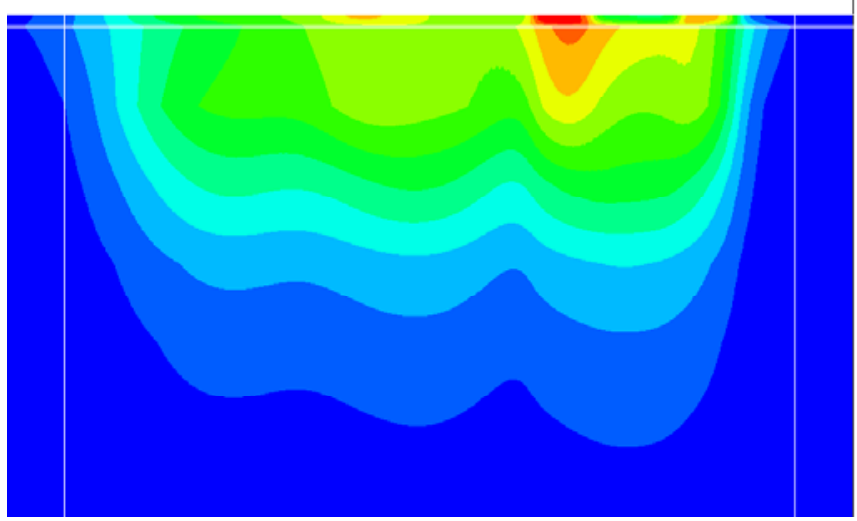

Color Index

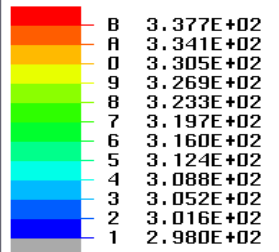

Fig. 4.4 Temperature contour plots of the plane through the cross section shown in Fig. 3.2 at the end of (a) $1.0 \mu \mathrm{s}$, (b) $2.0 \mu \mathrm{s}$, and (c) $3.0 \mu \mathrm{s}$. Dose is $6.5 \mu \mathrm{C} / \mathrm{cm}^{2}$. Current density is $6.5 \mathrm{~A} / \mathrm{cm}^{2}$. 
However, in the second simulation at a current density of $6.5 \mathrm{~A} / \mathrm{cm}^{2}$, the effect is apparent. In Fig. 4.3, the peak temperature at the end of the second flash is $349 \mathrm{~K}$ as compared with $339 \mathrm{~K}$ at the end of the first flash. The figure also shows how the residual heating from the first flash only affects part of the temperature profile of the second flash. This is observed as the red color tapers away to yellow. Finally, Fig. 4.4 shows that temperature profile of the second flash is extremely asymmetrical because of the heat diffusion from the first flash. Again, it can be seen that only the contours near the first flash are affected by the proximity heating effect, while those further away still resemble the profile of an unaffected flash. 


\section{Chapter 5}

\section{Model II: Results and Analysis}

\subsection{Visualization of Temperatures During Serpentine Writing}

Model II was designed to show that the FE models are capable of simulating complete patterns. Presented in Figs. $5.1-5.8$ are the temperature profiles at the surface of the resist at various points in the serpentine patterning scheme. The area within the red dotted lines represents the actively patterned area. Black dotted lines outline various flash locations and shape.

The surface temperature profile at the end of the $16^{\text {th }}$ flash, which is $31 \mu$ s into the simulation, is shown in Fig. 5.1. The e-beam has just completed patterning of the first row and will start on the second row by moving to the black outlined square above and to the left. The previous flash is shown as the outlined square to the right. The peak temperature is $343 \mathrm{~K}$.

The surface temperature profile at the end of the $17^{\text {th }}$ flash, $33 \mu$ s into the simulation, is shown in Fig. 5.2. It is the first flash to be written in the second row. Noticeable is the thermal tail that is still dissipating from the writing of the row 6 below. This affects the peak temperature of the resist, which is $344.6 \mathrm{~K}$.

Corresponding to the profile at the end of the $18^{\text {th }}$ flash, $35 \mu$ s into the simulation, is Fig. 5.3. This is the second flash of the second row. The peak temperature here is $346.3 \mathrm{~K}$. Higher peak temperatures are seen here because the 
close proximity of the previously flashed areas causes the dissipating heat to raise the temperature of the area currently being exposed. After the beam rounds this corner however, the effect of previous exposures is diminished and the peak temperatures begin to decline until it reaches the end of the row.

The last flash of the second row is shown in Fig. 5.4. This corresponds to the $32^{\text {nd }}$ flash, $63 \mu$ s into the simulation. The peak temperature has decreased to $343.6 \mathrm{~K}$. This is higher than the temperature at the end of the first row of writing which was $343 \mathrm{~K}$. The small temperature rise that occurs from one row to the next is called global heating, and is caused by the dissipation of energy introduced by the e-beam into the substrate. This rise is relatively small because of the extreme mass of the substrate.

The remaining plots, Figs. 5.5 - 5.8, for the continued patterning of the remaining rows show the same basic trends that occurred from writing the first two rows. Not only are the maximum temperatures always seen at the same location as the beam rounds the corner and moves from one row to the next, but the maximum temperatures are also fairly similar to one another. 


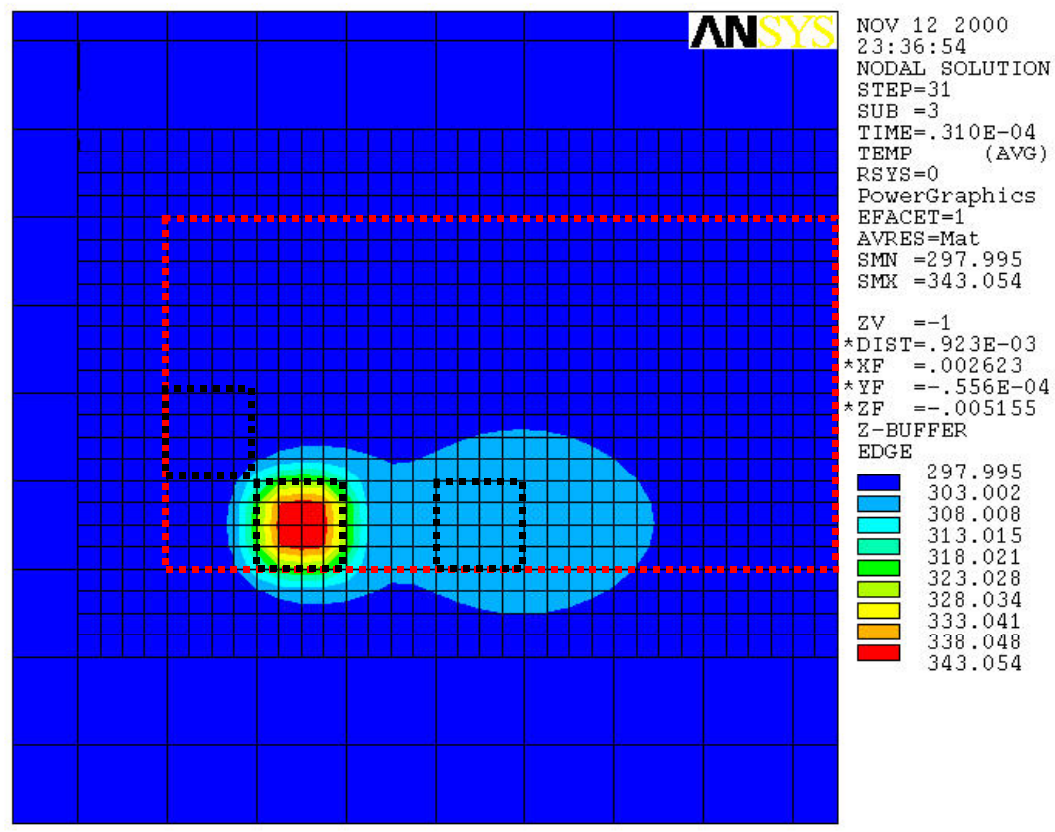

Fig. 5.1 Temperature contour plots on the top layer of the resist for the $16^{\text {th }}$ flash of serpentine writing, $31 \mu \mathrm{s}$ into the simulation.

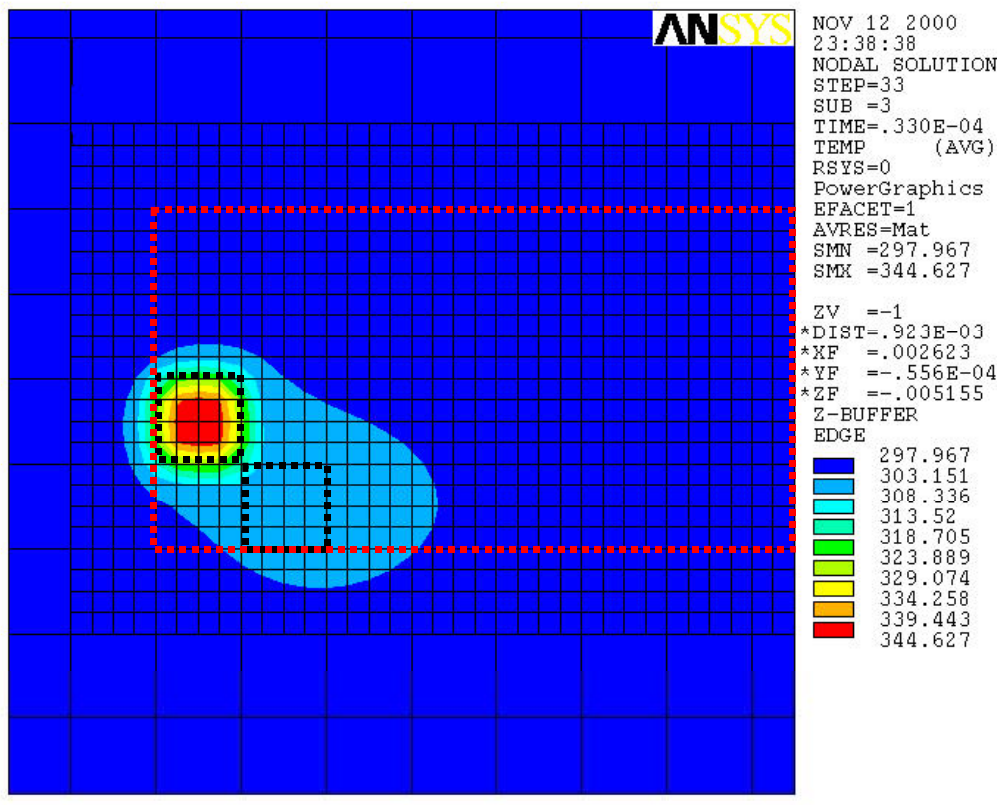

Fig. 5.2 Temperature contour plots on the top layer of the resist for the $17^{\text {th }}$ flash of serpentine writing, $33 \mu \mathrm{s}$ into the simulation. 


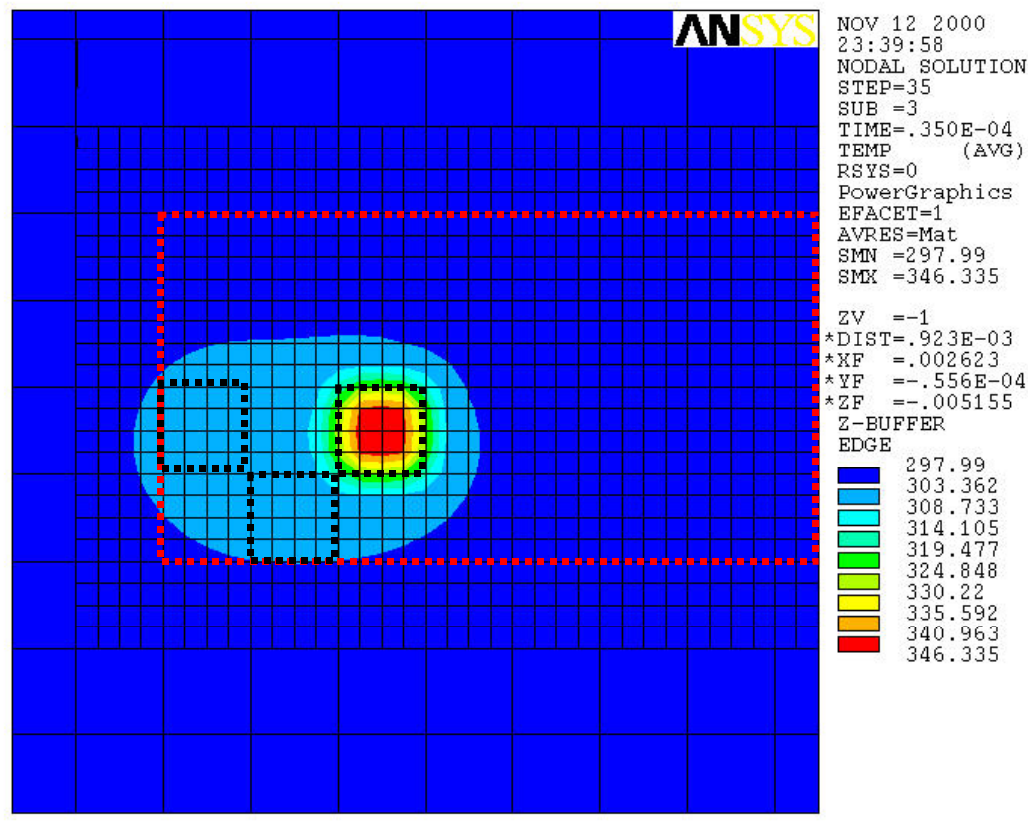

Fig. 5.3 Temperature contour plots on the top layer of the resist for the $18^{\text {th }}$ flash of serpentine writing, $35 \mu \mathrm{s}$ into the simulation.

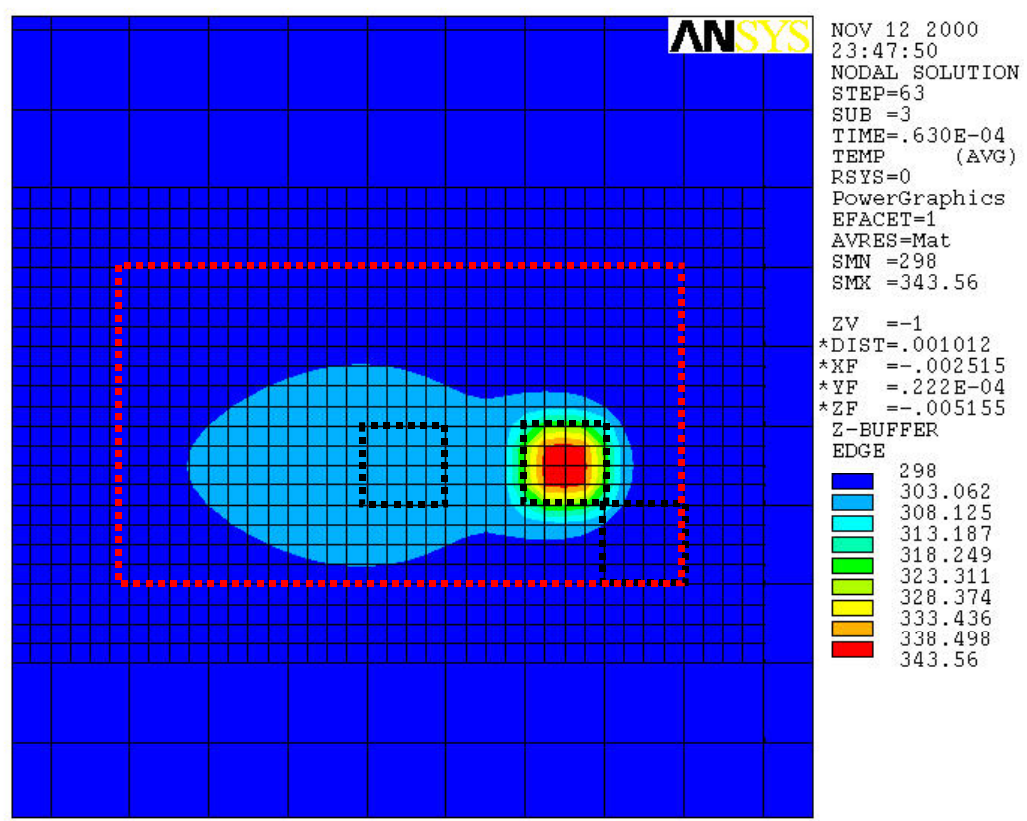

Fig. 5.4 Temperature contour plots on the top layer of the resist for the $32^{\text {nd }}$ flash of serpentine writing, $63 \mu \mathrm{s}$ into the simulation. 


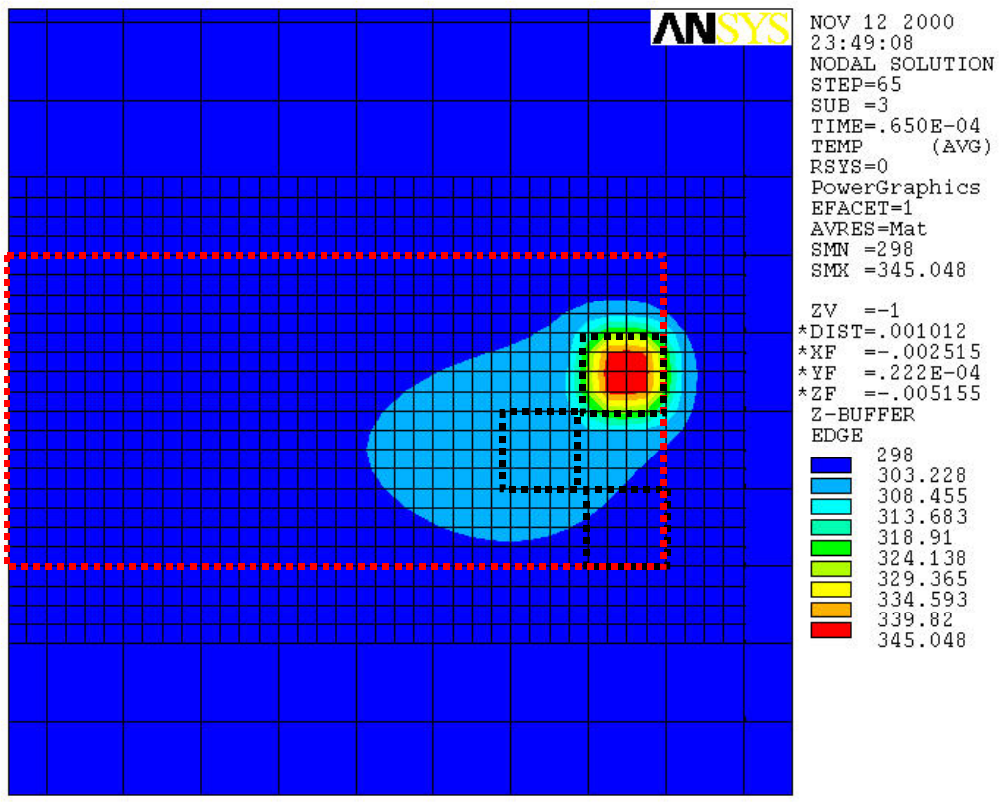

Fig. 5.5 Temperature contour plots on the top layer of the resist for the $33^{\text {rd }}$ flash of serpentine writing, $65 \mu \mathrm{s}$ into the simulation.

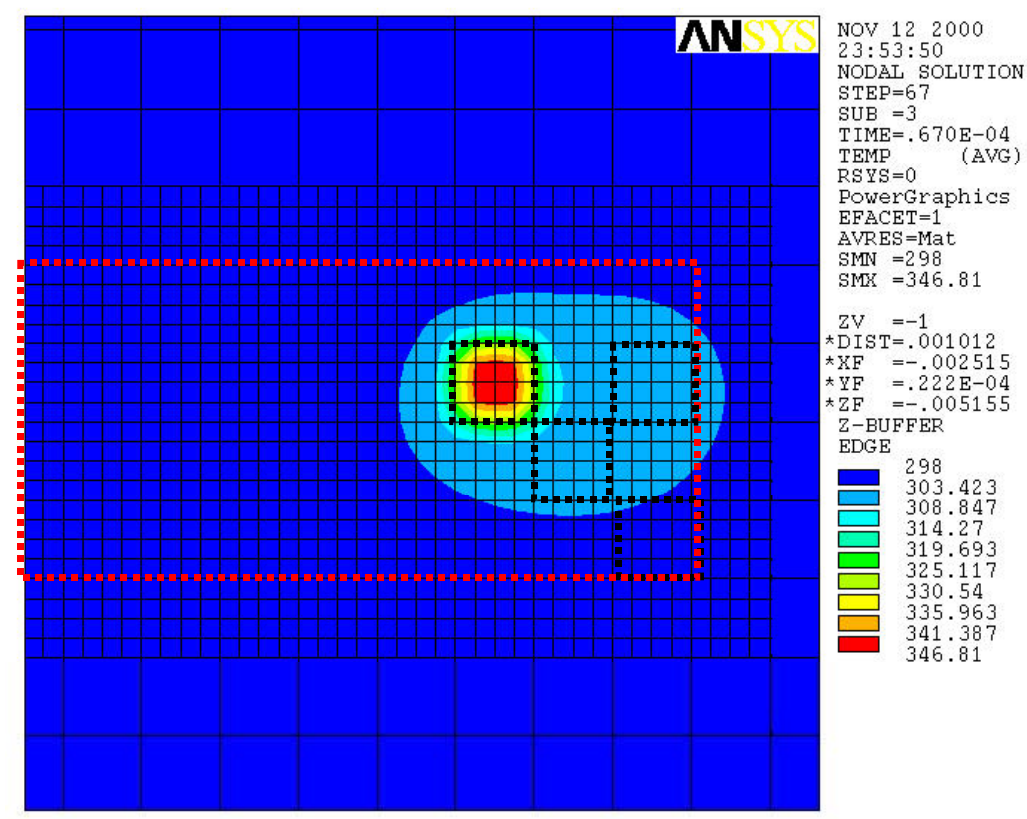

Fig. 5.6 Temperature contour plots on the top layer of the resist for the $34^{\text {th }}$ flash of serpentine writing, $67 \mu \mathrm{s}$ into the simulation. 


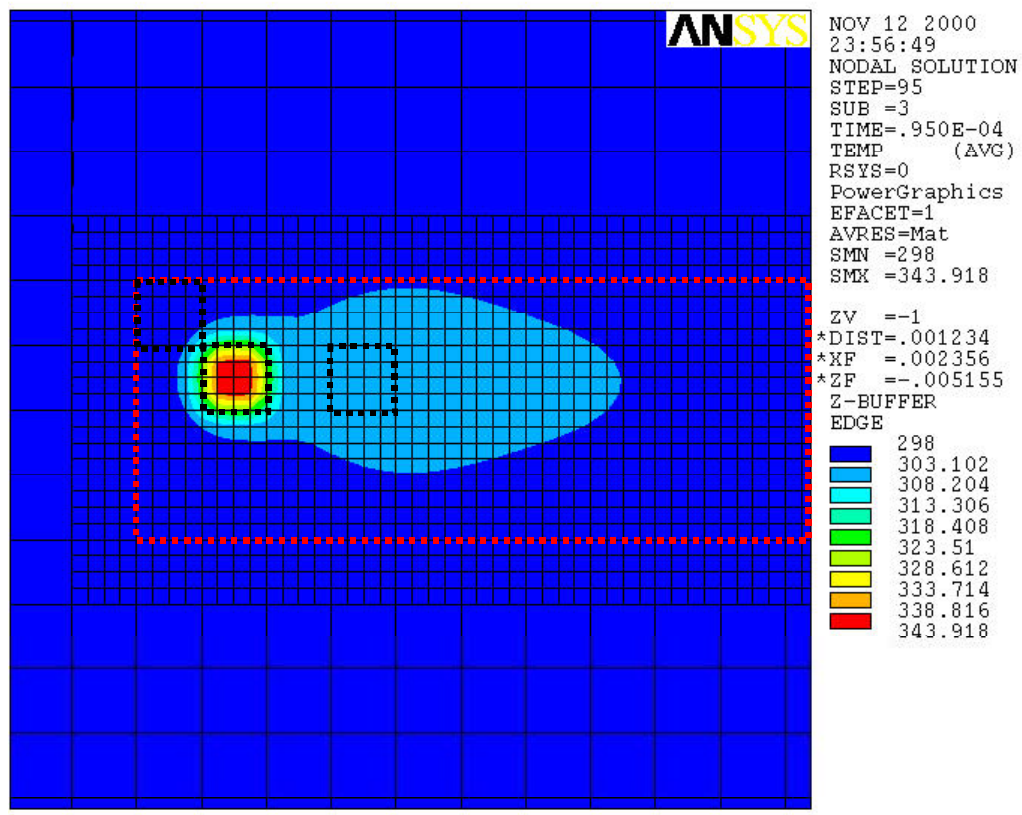

Fig. 5.7 Temperature contour plots on the top layer of the resist for the $48^{\text {th }}$ flash of serpentine writing, $95 \mu \mathrm{s}$ into the simulation.

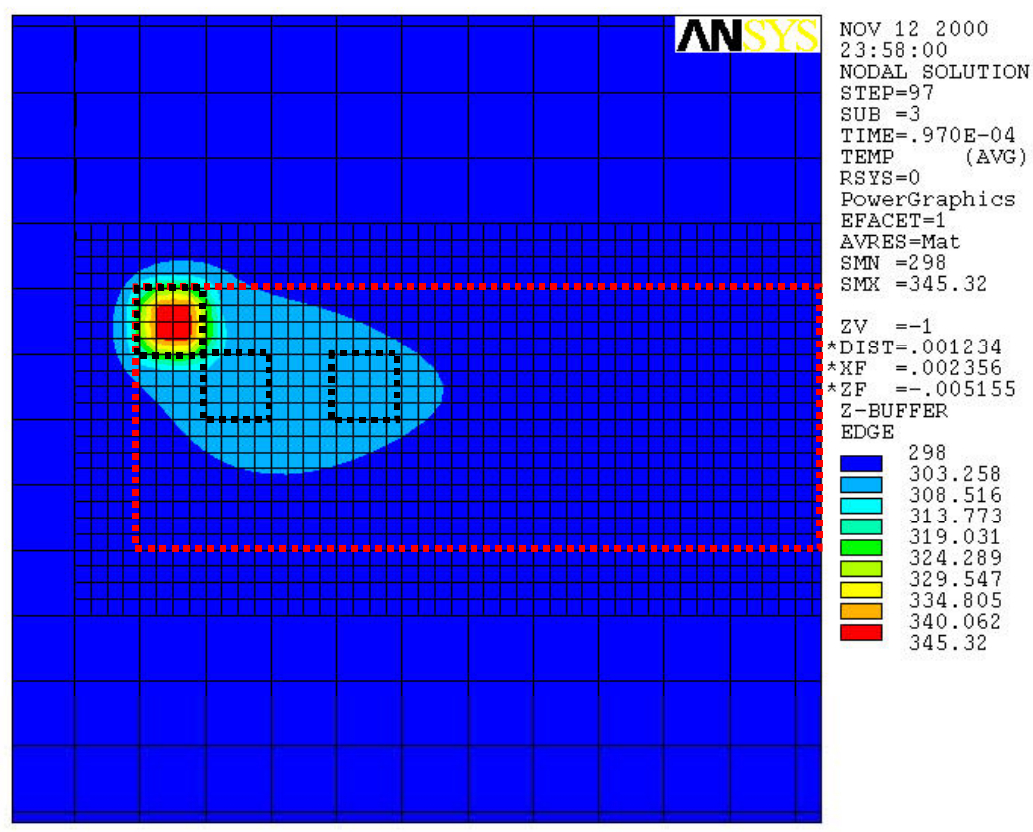

Fig. 5.8 Temperature contour plots on the top layer of the resist for the $49^{\text {th }}$ flash of serpentine writing, $97 \mu \mathrm{s}$ into the simulation. 


\subsection{Visualization of Temperatures During Unidirectional Writing}

Presented in Figs. 5.9 - 5.13 are the temperature profiles at the surface of the resist at various points in the unidirectional patterning scheme. Again, the area within the red dotted lines represents the actively patterned area and black dotted lines outline various flash locations and shape.

The surface temperature profile at the end of the $17^{\text {th }}$ flash, $33 \mu$ s into the simulation, is shown in Fig. 5.9. The e-beam has completed patterning of the first row, reset itself to the beginning of the second row, and exposed the first shape in the second row. The peak temperature is $342.2 \mathrm{~K}$. In Fig. 5.9 it is possible to see the residual heat from the patterning of the first row in the left-had side of the figure. In a serpentine patterning strategy, as discussed in section 5.1, this residual heat would affect the next few flashes of the second row. In this unidirectional strategy the last flash of the first row and the first flashes of the second row are so far apart that there is virtually no crossover effect from one row to the next. The global heating effect is still present but relatively minute for this case. A closer view of the currently written spot is shown in Fig. 5.10. The highly symmetrical contours signify that there were no outside influences on the flash.

The surface temperature profile at the end of the $49^{\text {th }}$ flash, $97 \mu$ s into the simulation, is shown in Fig. 5.11. It is the first flash to be written in the fourth row. The peak temperature at the end of this flash is $342.9 \mathrm{~K}$. As mentioned above, the overall temperatures for this flash are slightly higher than for Fig. 5.9 because of global heating. Otherwise, the two figures are very similar. 
In Figs. 5.12 and 5.13, corresponding to the $32^{\text {nd }}$ (63 $\mu$ s into the simulation) and $64^{\text {th }}(127 \mu$ s into the simulation), respectively, the similarities from one row to the next can, again, be seen.

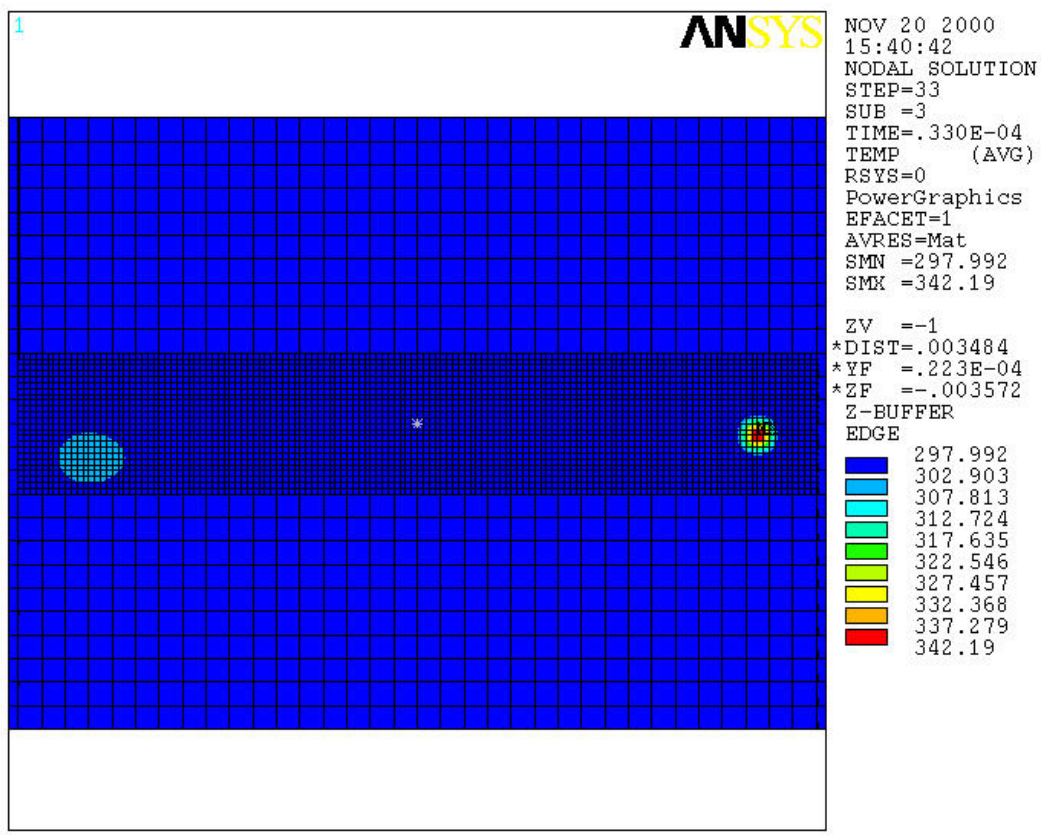

Fig. 5.9 Farfield view of temperature contour plots on the top layer of the resist for the $17^{\text {th }}$ flash of unidirectional writing, $33 \mu$ s into the simulation. 


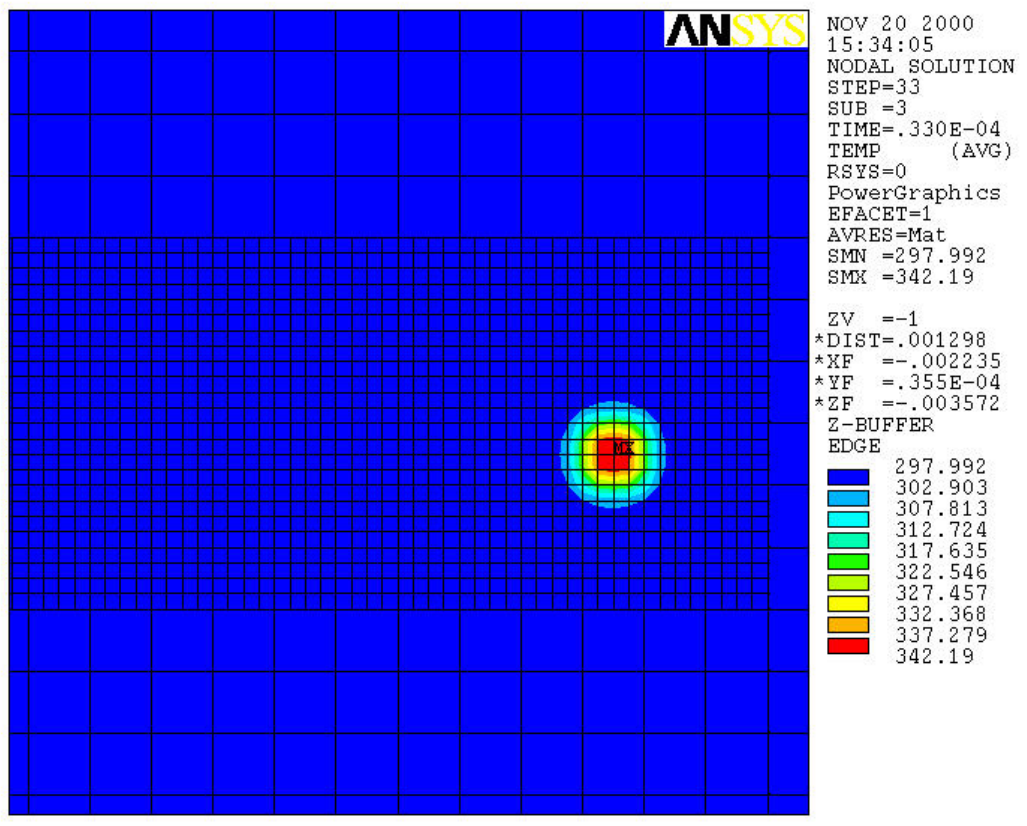

Fig. 5.10 Nearfield view of temperature contour plots on the top layer of the resist for the $17^{\text {th }}$ flash of unidirectional writing, $33 \mu$ s into the simulation.

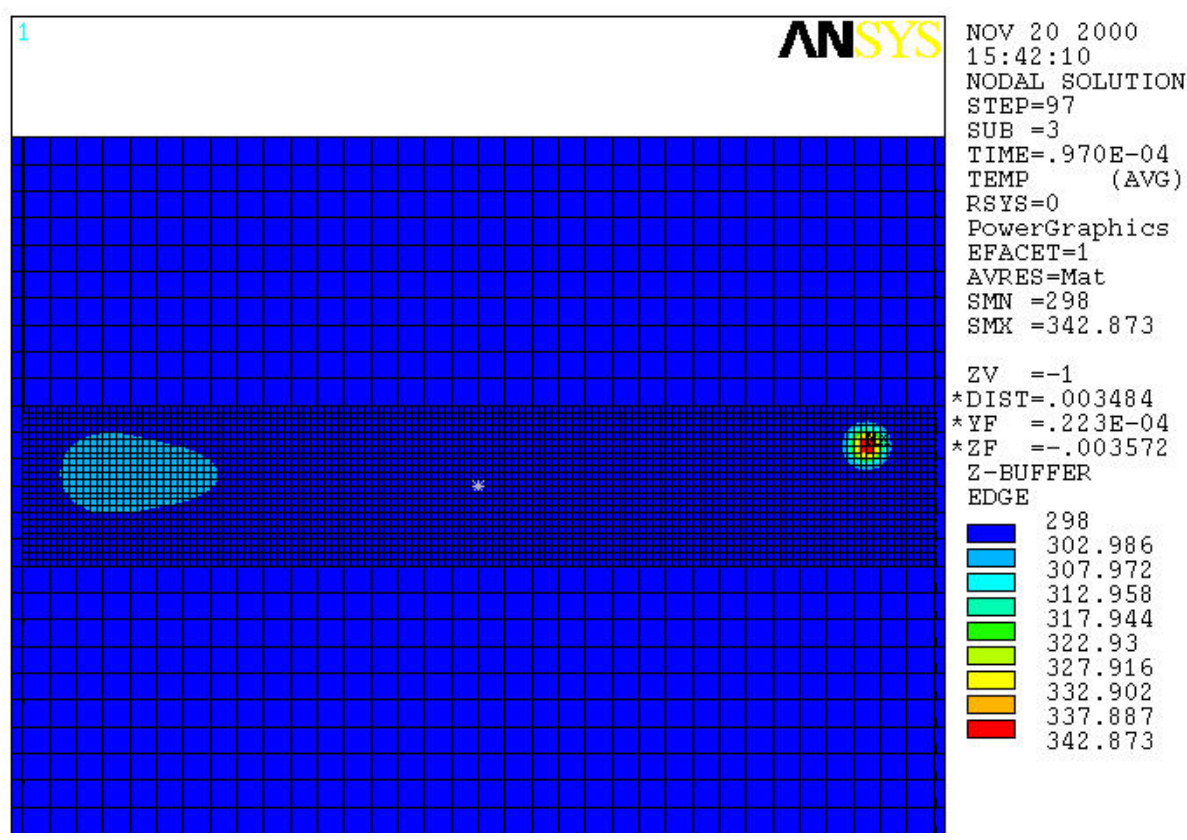

Fig. 5.11 Farfield view of temperature contour plots on the top layer of the resist for the $49^{\text {th }}$ flash of unidirectional writing, $97 \mu$ s into the simulation. 


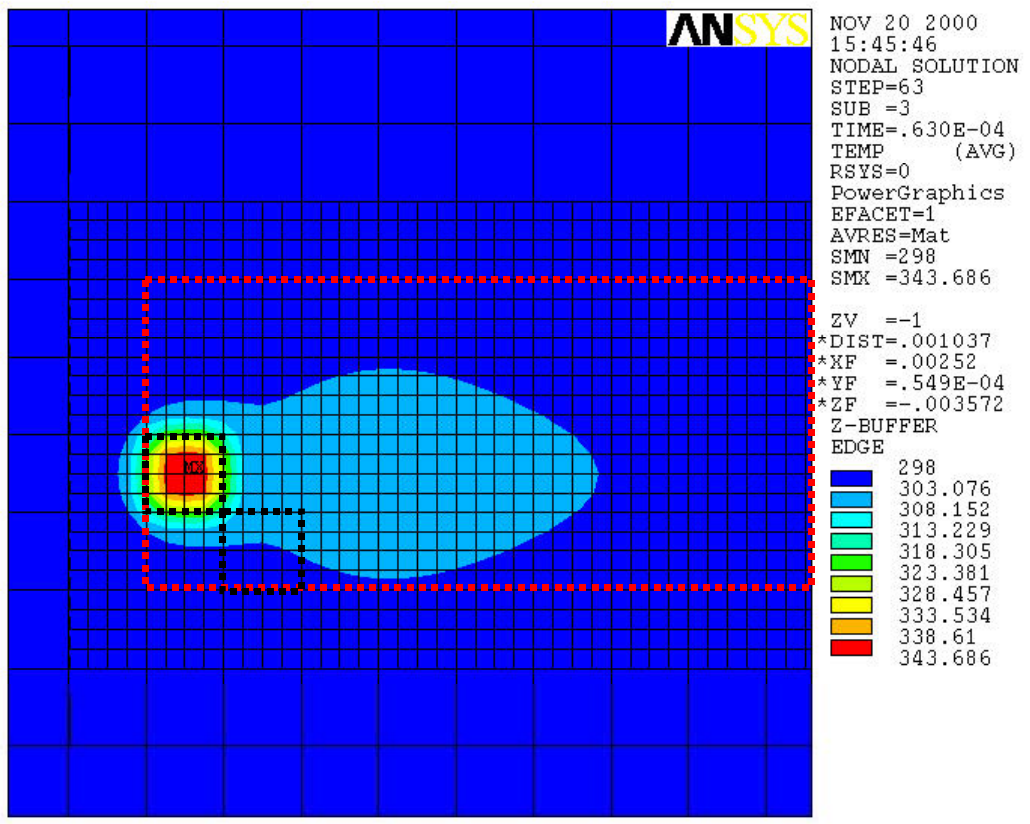

Fig. 5.12 Temperature contour plots on the top layer of the resist for the $32^{\text {nd }}$ flash of unidirectional writing, $63 \mu$ s into the simulation.

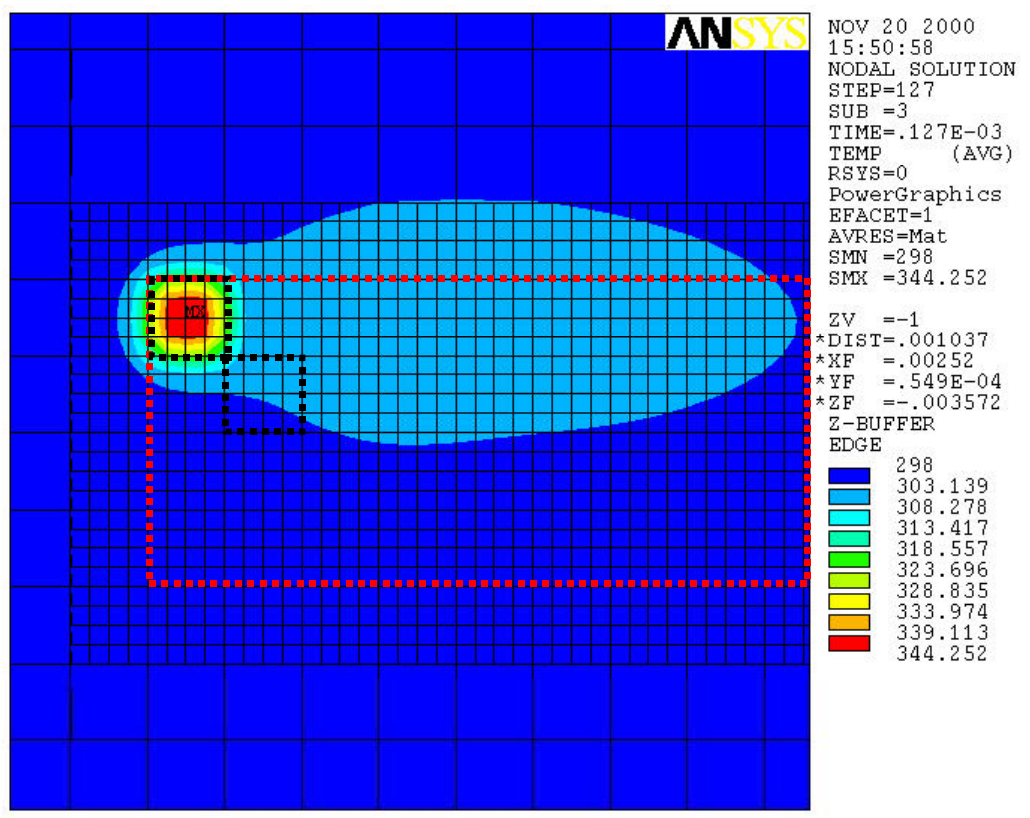

Fig. 5.13 Temperature contour plots on the top layer of the resist for the $64^{\text {th }}$ flash of unidirectional writing, $127 \mu$ s into the simulation. 


\subsection{Temperature Histories at Various Points in the Patterned Subfield}

The plots and results shown in the above sections are good visualization tools and help in understanding how the heat dissipates during patterning. Unfortunately, it is very hard to use the information in these figures to perform calculations needed to predict the sensitivity changes in the resist, which is the ultimate goal. Specifically, what is desired is to compare the integrated temperature effects of a point in the patterned area to the resist profile of the same point in an experiment. It may then be possible to see the extent to which the temperature affects the sensitivity.

To present the results in a more useful format, the temperature histories at various points were plotted as a function of time. Shown below in Fig. 5.14 is a map of the locations of these points. The yellow boxes represent areas of direct flash exposure. The circled numbers indicate the points at which the temperature histories were extracted. Each of the following plots in Figs. 5.15 - 5.30 corresponds to one of these mapped points. Plots were constructed for both the serpentine and unidirectional writing schemes. 

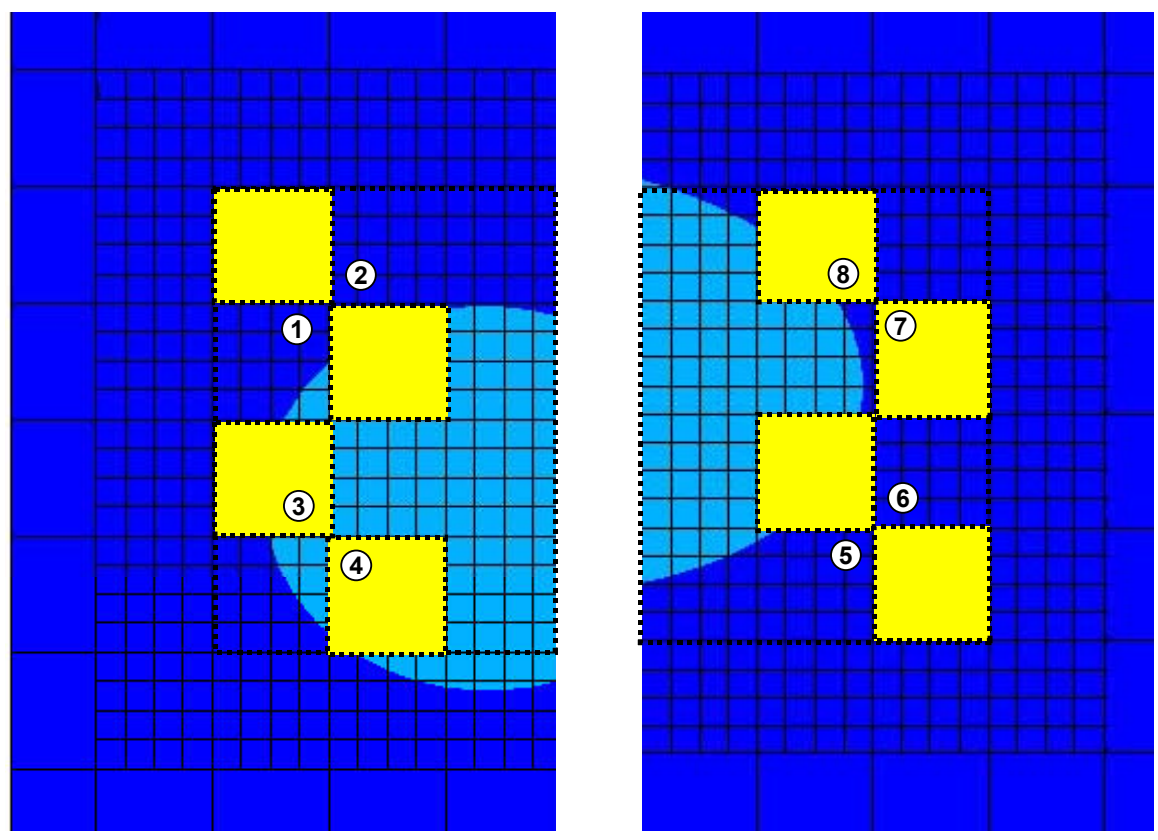

Fig. 5.14 Points at which temperature history plots for Figs. 5.15 - 5.30 were generated.

Analyzing Fig. 5.15, the first features that can be seen are the two peaks that occur at approximately 32 and $96 \mu$ s. The first peak corresponds to the patterning of the first two rows. In the serpentine patterning, the field in which Point 4 is located is exposed and then the field in which Point 3 is located is exposed. The patterning then continues on to the right from Point 3. The ragged peak implies that multiple exposures affect the temperature at this point. Before the temperature spike from one exposure is allowed to dissipate, another flash occurs and creates a secondary temperature spike. The peak seen at Point 1 is most probably caused by a combination of three flashes: the one containing Point 3, the one containing Point 4, and the one following Point 3. The same effect is seen at $96 \mu$ s. 


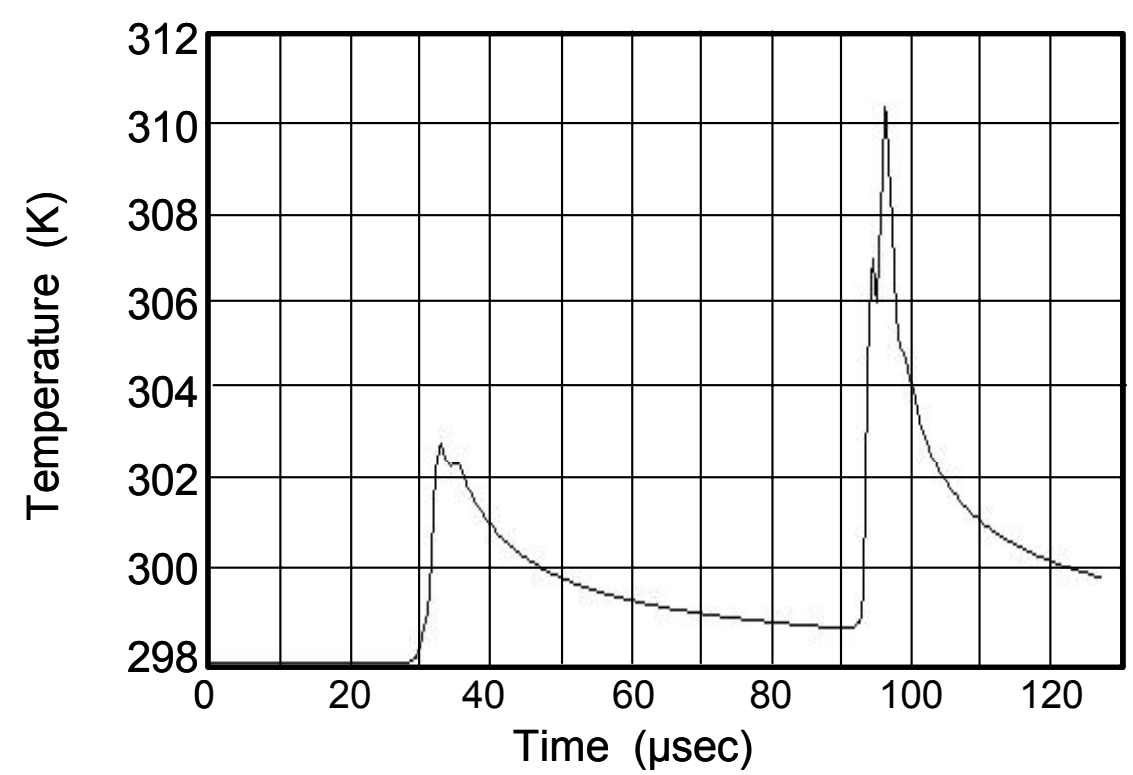

Fig. 5.15 Temperature history corresponding to Point 1 in Fig. 5.14 for serpentine writing.

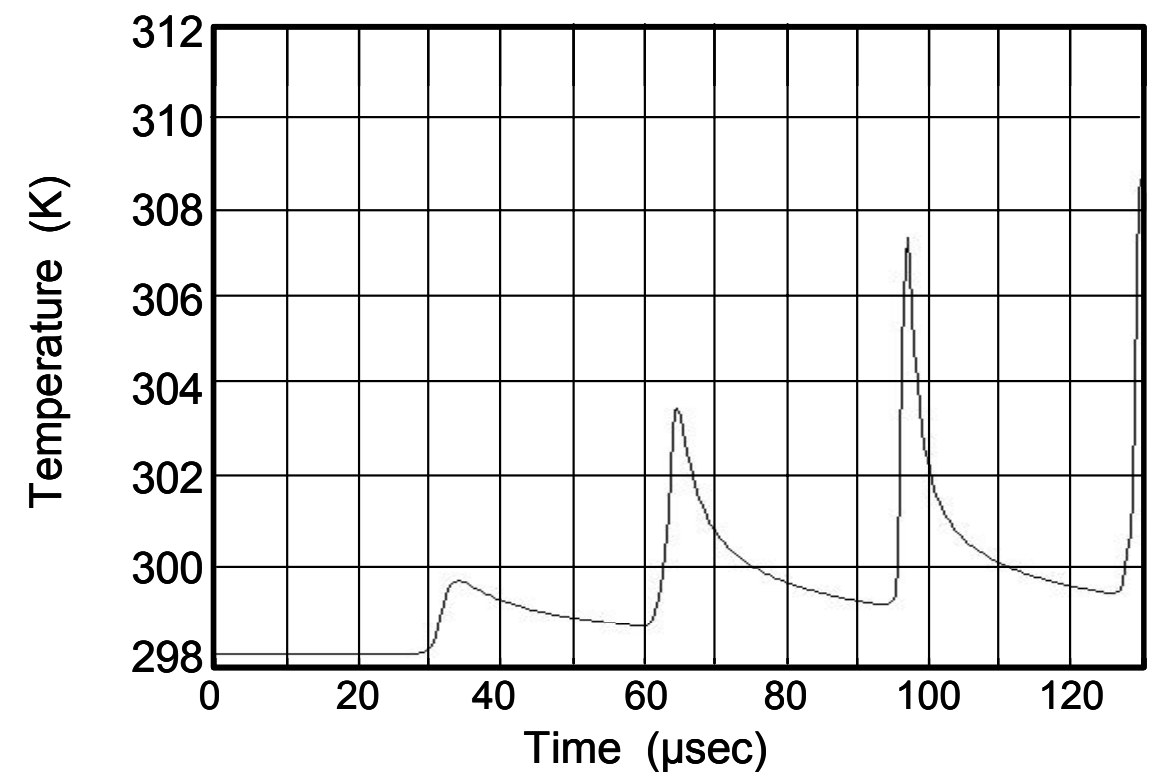

Fig. 5.16 Temperature history corresponding to Point 1 in Fig. 5.14 for unidirectional writing. 
Comparing Figs. 5.15 and 5.16, the first noticeable difference is that there are more peaks in Fig. 5.16, and they are devoid of any inconsistencies as seen in the case for serpentine writing, Fig. 5.15. Each peak has a definite high point and follows an expected thermal decay pattern. These definitive peaks occur because in the unidirectional writing strategy there are no exposures that occur in close proximity to one another within small time intervals. These points, at which the e-beam turns the corner from one row to the next in serpentine patterning, have been eliminated. The exposures, which in the serpentine strategy affected one another, have now been separated due to the resetting of e-beam to the beginning of the next line.

The remaining plots all exhibit similar features as those described above, and the explanation as to their origins remains the same. The plots for Points 3, 4, 7, and 8 differ slightly from the plots for Points $1,2,5$, and 6 , because they are located within the flashed area instead of outside. They still exhibit the multiple peaks due to the localized heating however. 


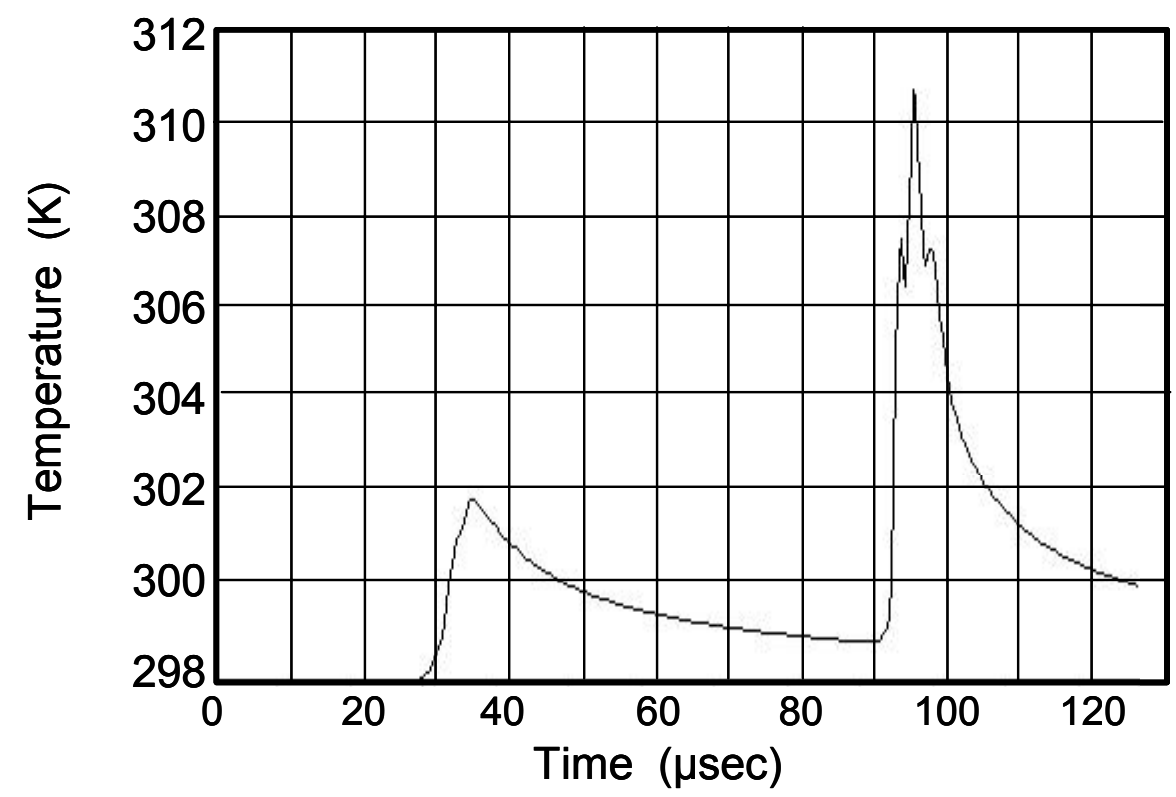

Fig. 5.17 Temperature history corresponding to Point 2 in Fig. 5.14 for serpentine writing.

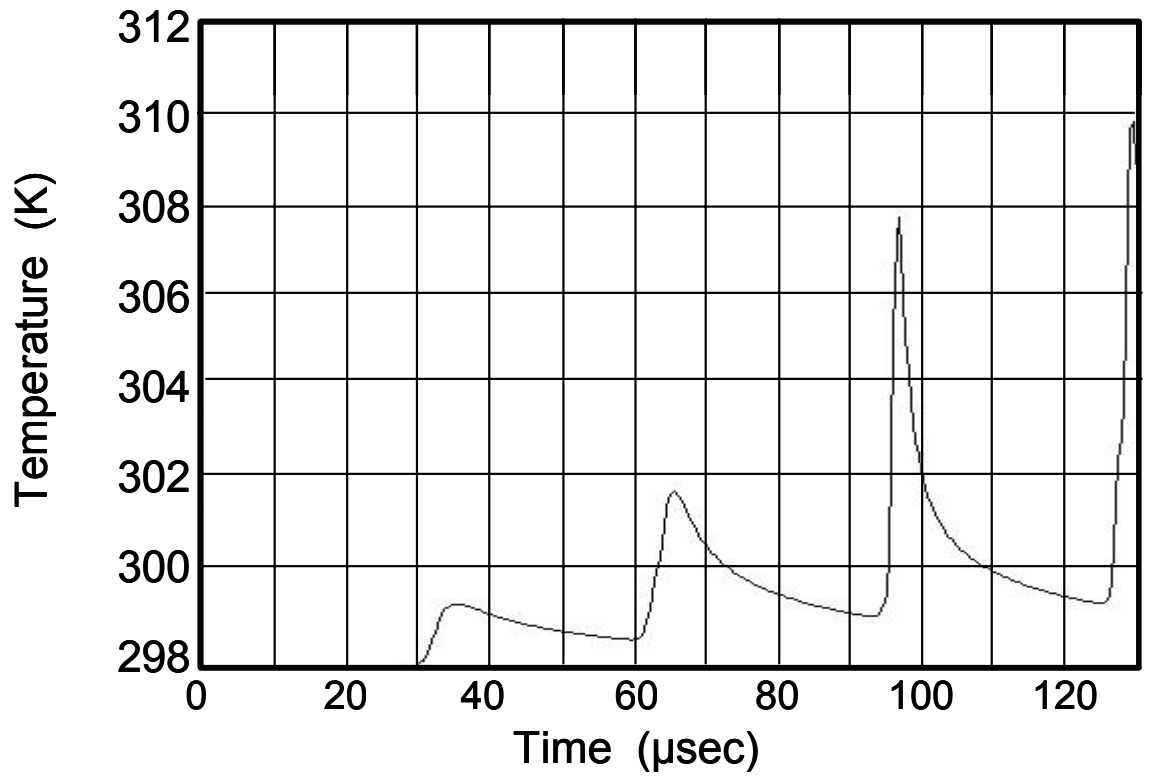

Fig. 5.18 Temperature history corresponding to Point 2 in Fig. 5.14 for unidirectional writing. 


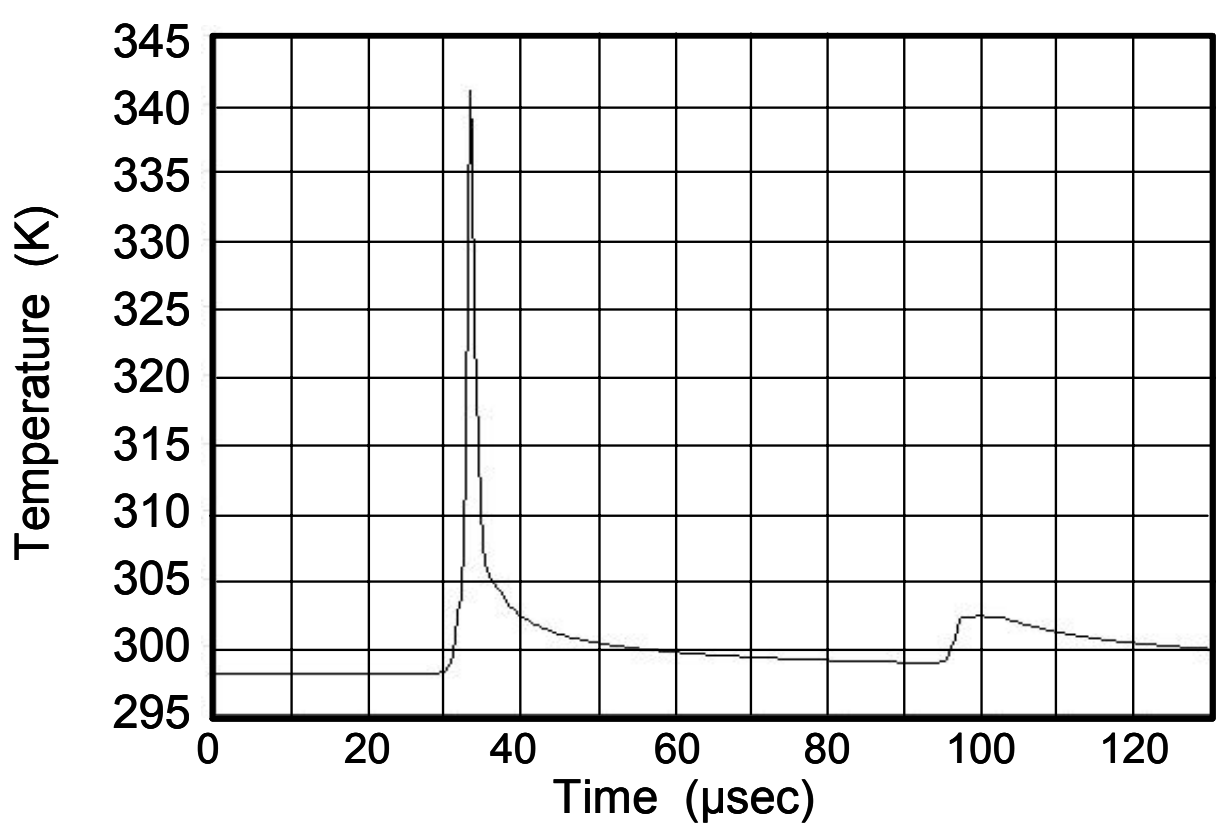

Fig. 5.19 Temperature history corresponding to Point 3 in Fig. 5.14 for serpentine writing.

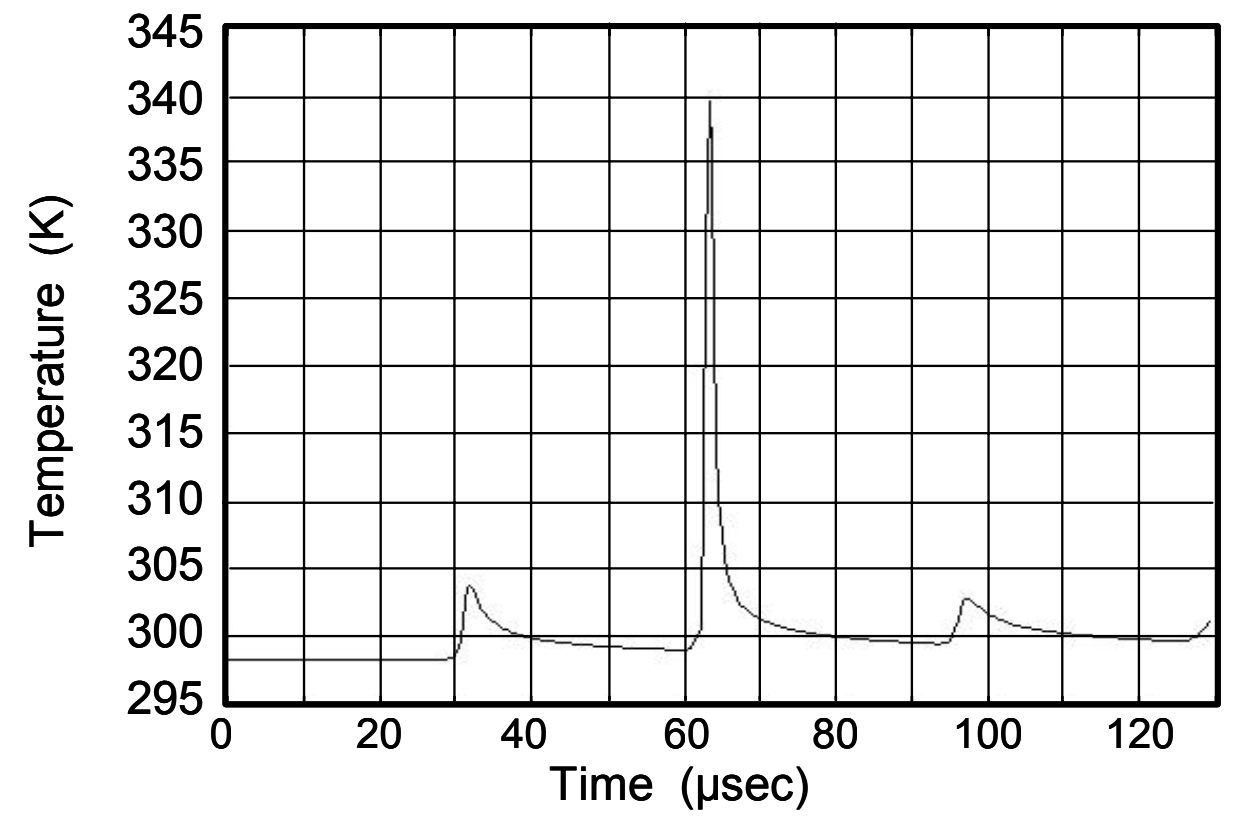

Fig. 5.20 Temperature history corresponding to Point 3 in Fig. 5.14 for unidirectional writing. 


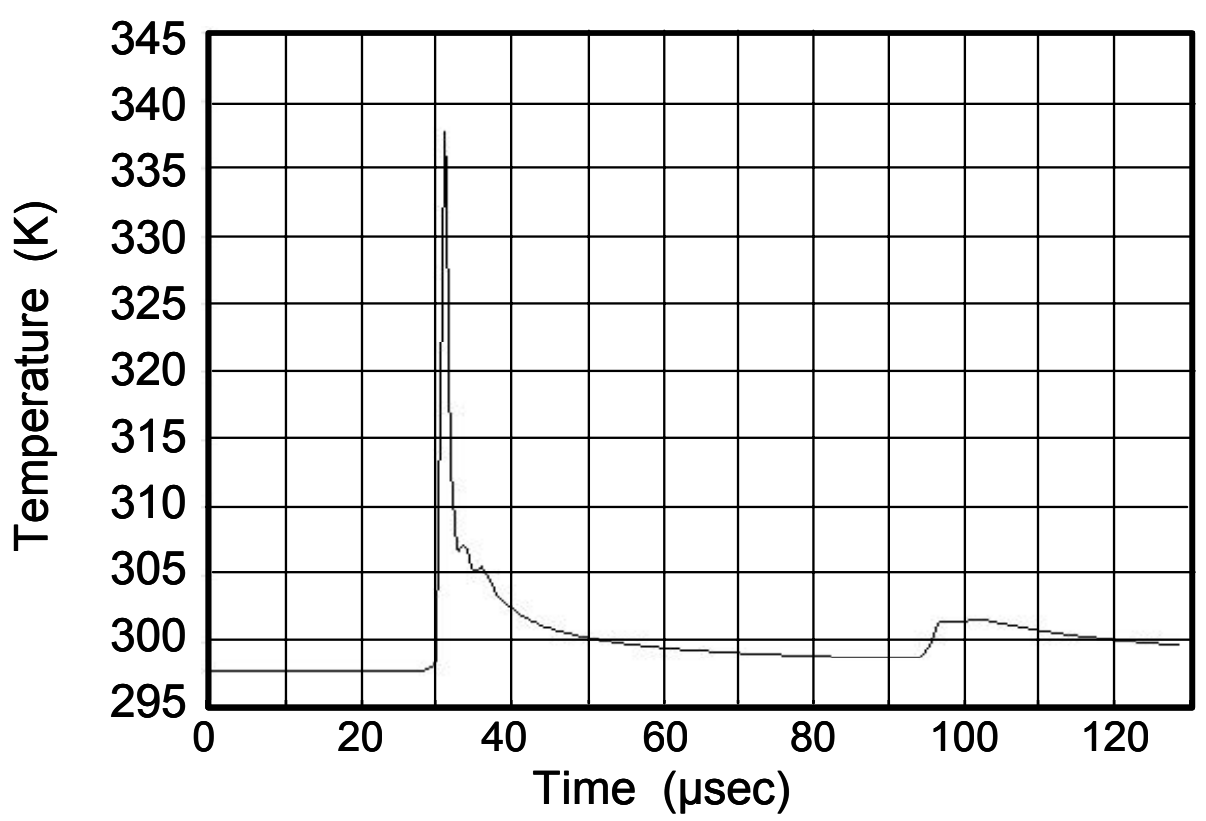

Fig. 5.21 Temperature history corresponding to Point 4 in Fig. 5.14 for serpentine writing.

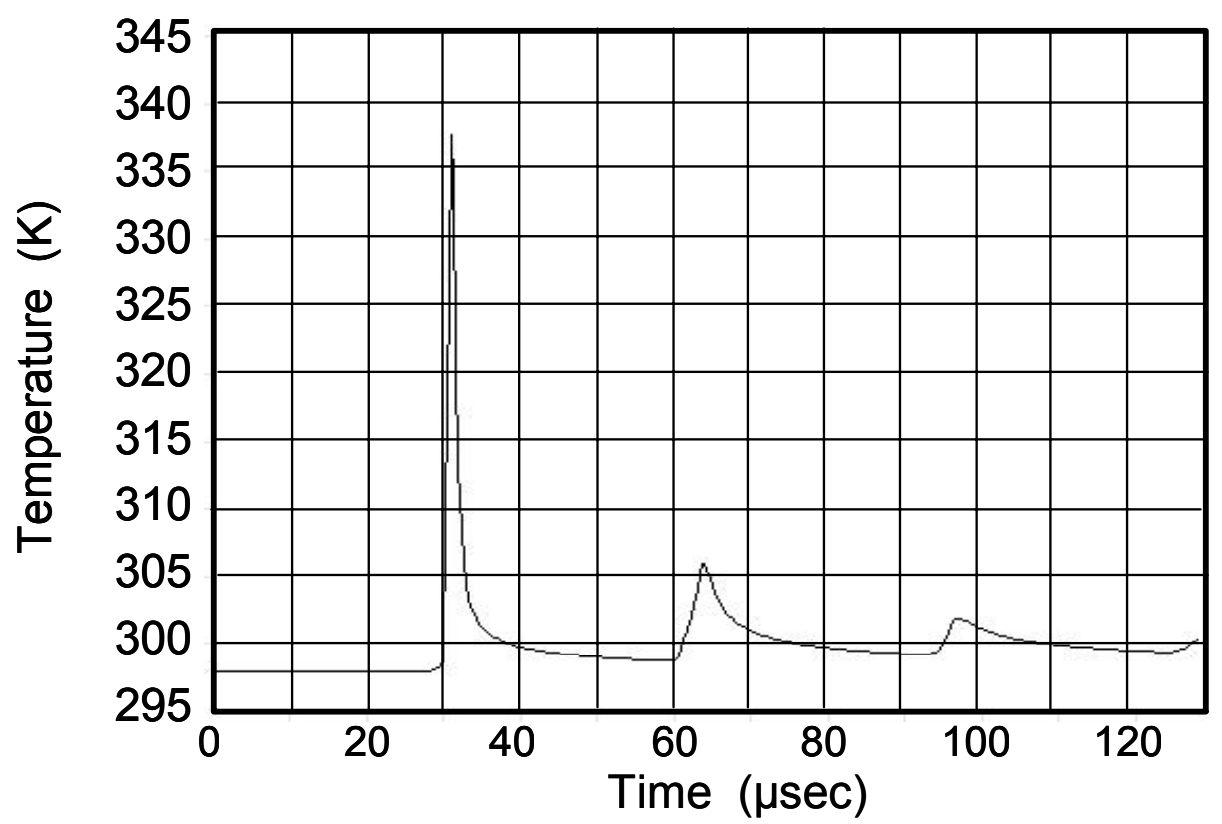

Fig. 5.22 Temperature history corresponding to Point 4 in Fig. 5.14 for unidirectional writing. 


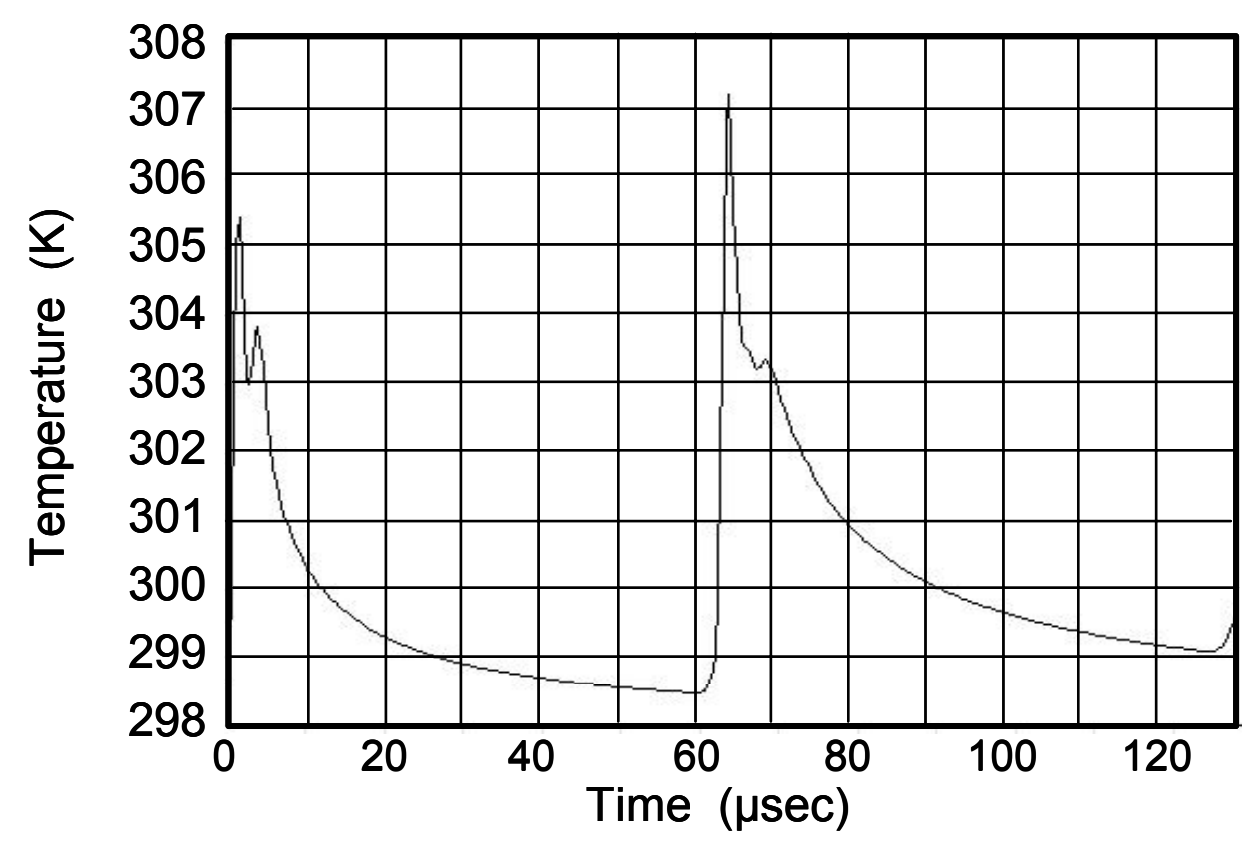

Fig. 5.23 Temperature history corresponding to Point 5 in Fig. 5.14 for serpentine writing.

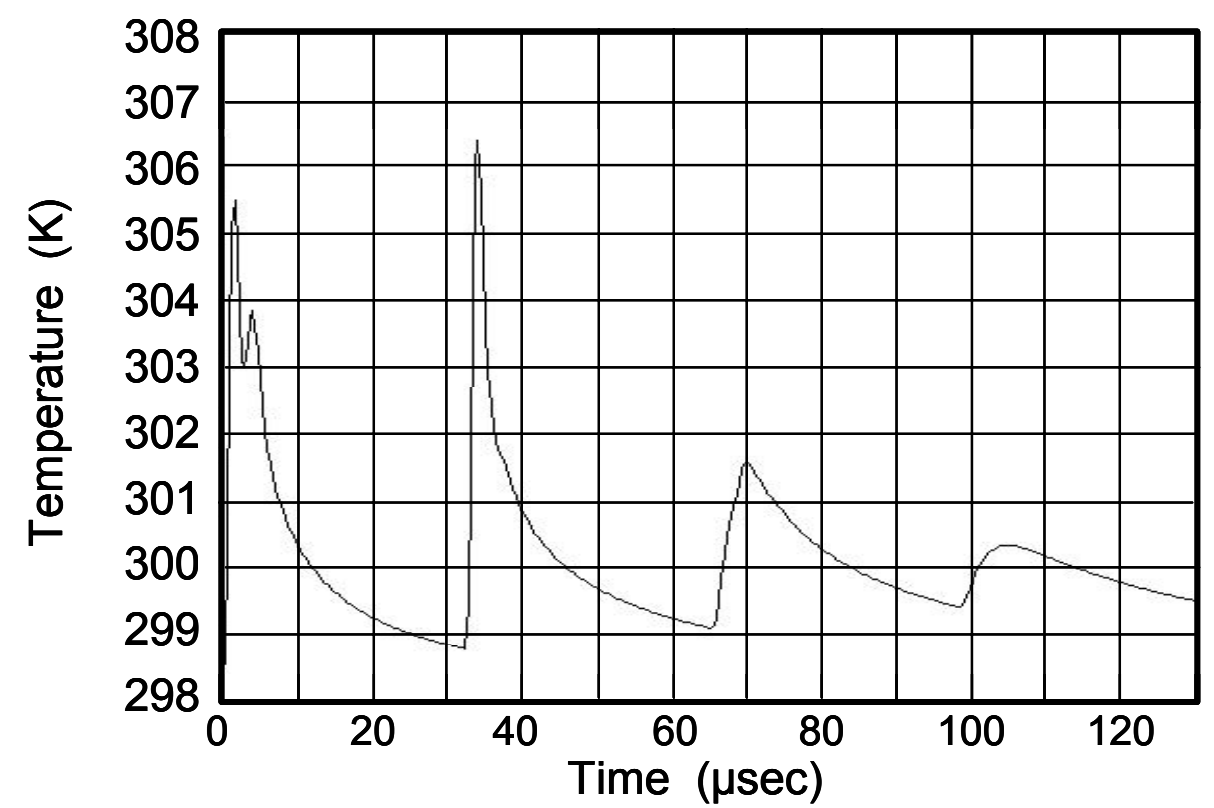

Fig. 5.24 Temperature history corresponding to Point 5 in Fig. 5.14 for unidirectional writing. 


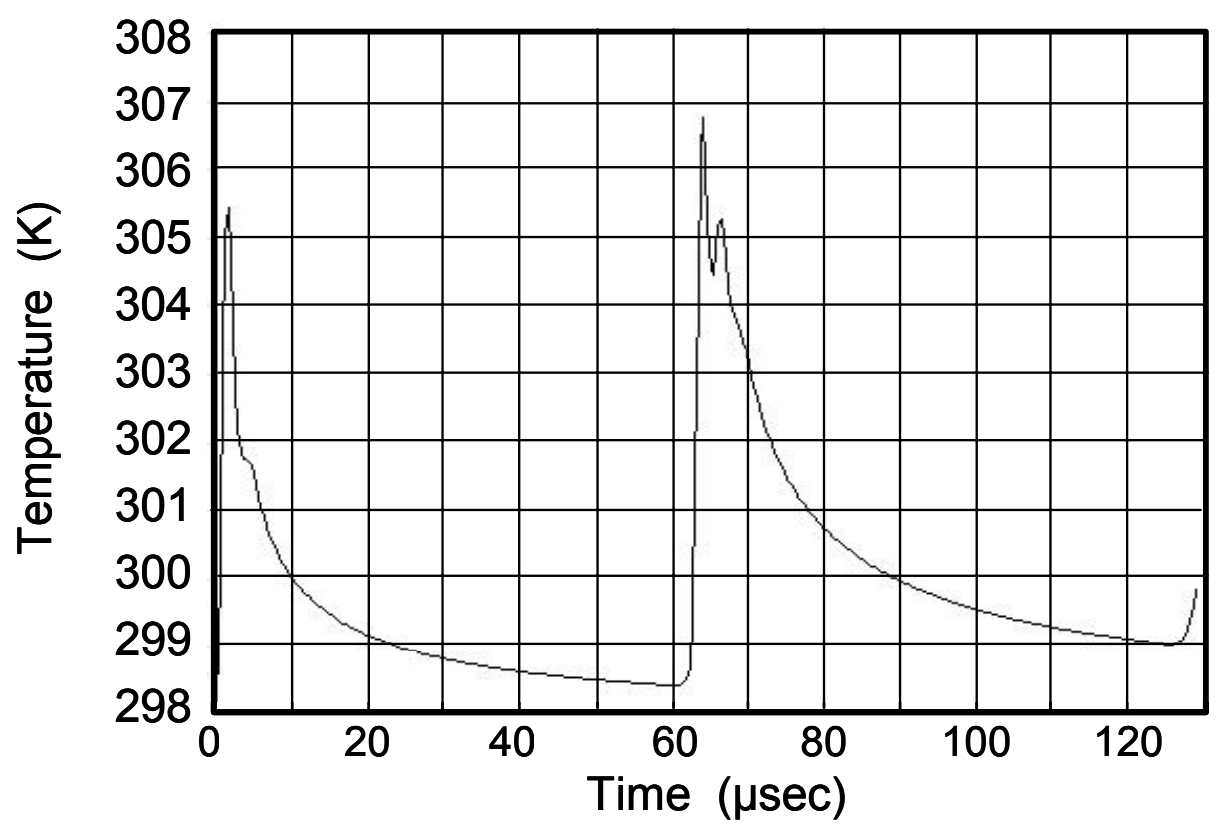

Fig. 5.25 Temperature history corresponding to Point 6 in Fig. 5.14 for serpentine writing.

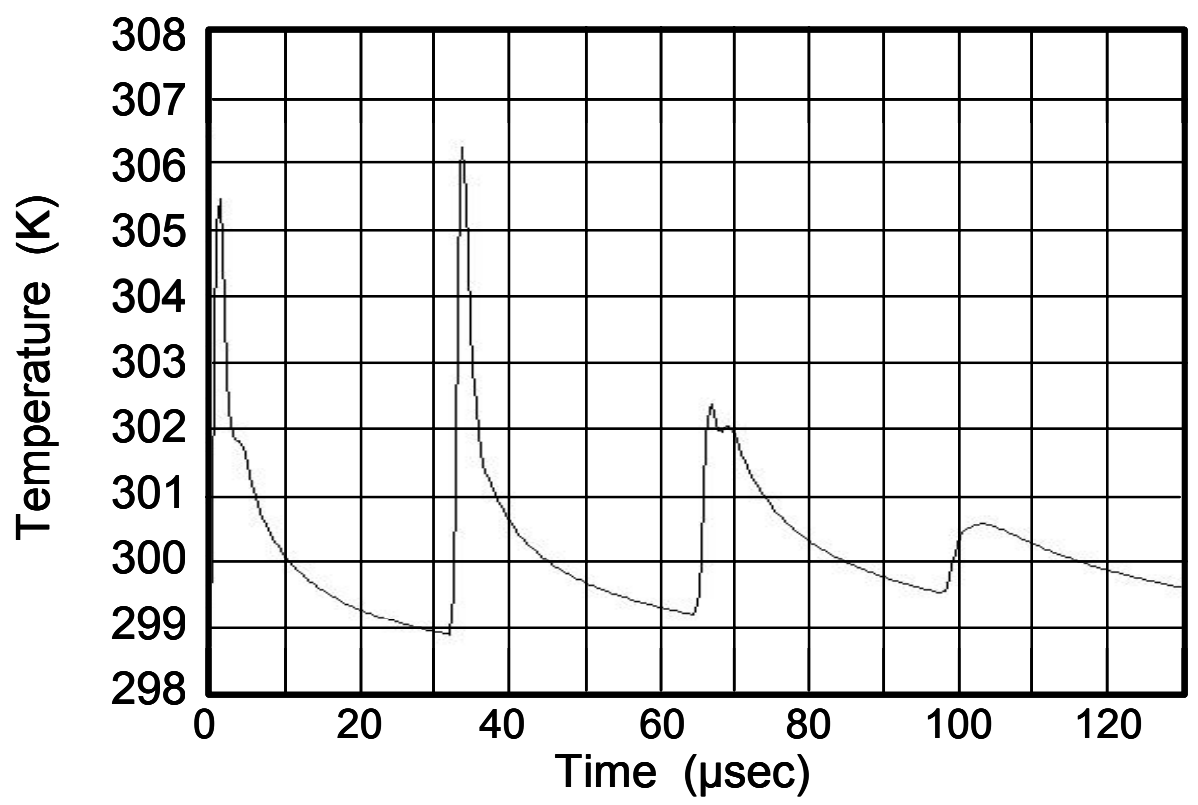

Fig. 5.26 Temperature history corresponding to Point 6 in Fig. 5.14 for unidirectional writing. 


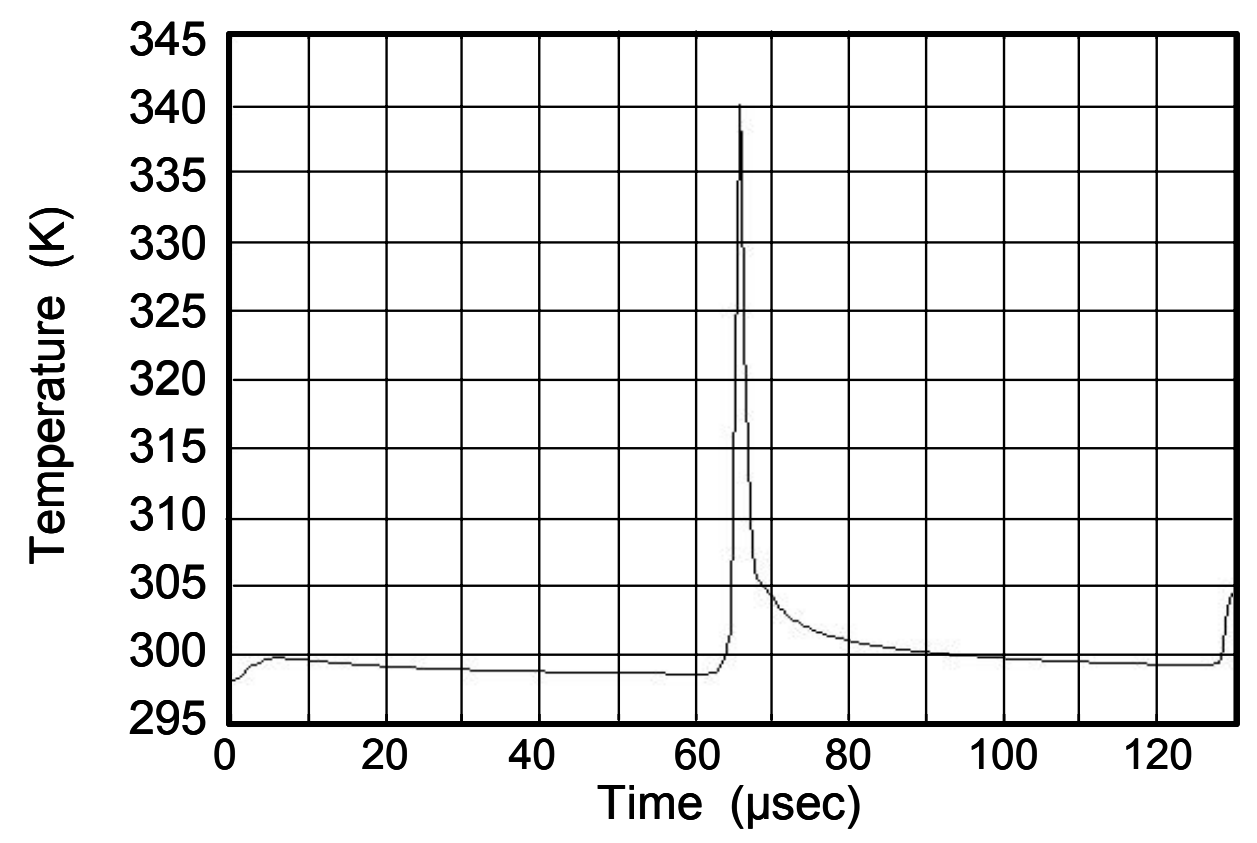

Fig. 5.27 Temperature history corresponding to Point 7 in Fig. 5.14 for serpentine writing.

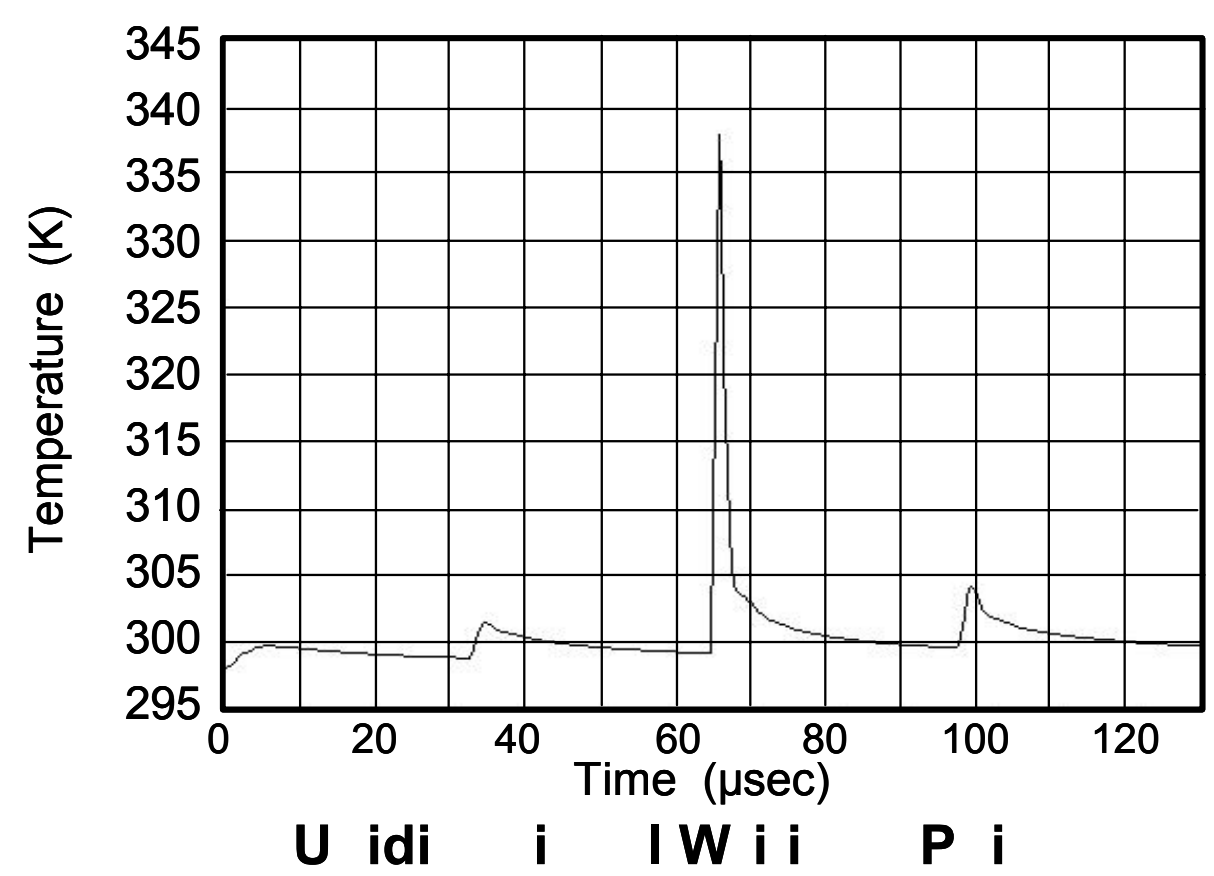

Fig. 5.28 Temperature history corresponding to Point 7 in Fig. 5.14 for unidirectional writing. 


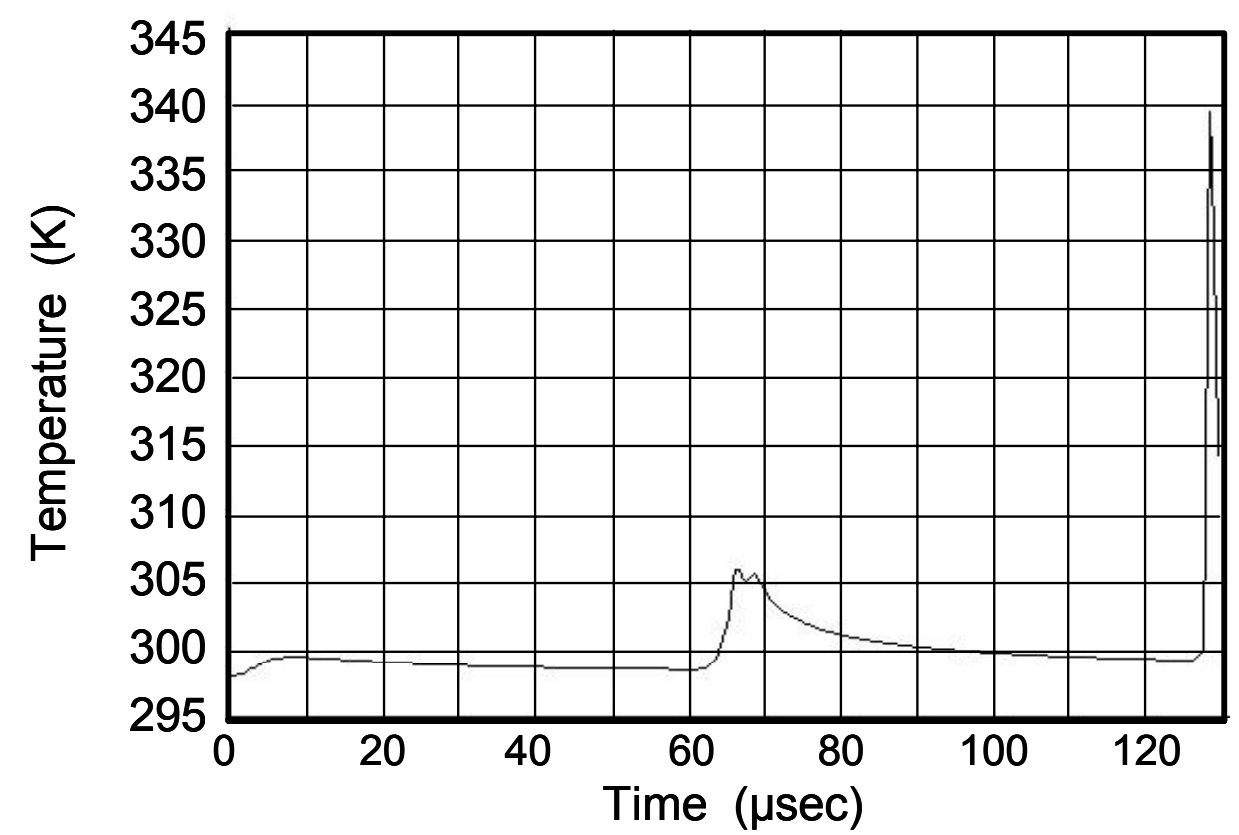

Fig. 5.29 Temperature history corresponding to Point 8 in Fig. 5.14 for serpentine writing.

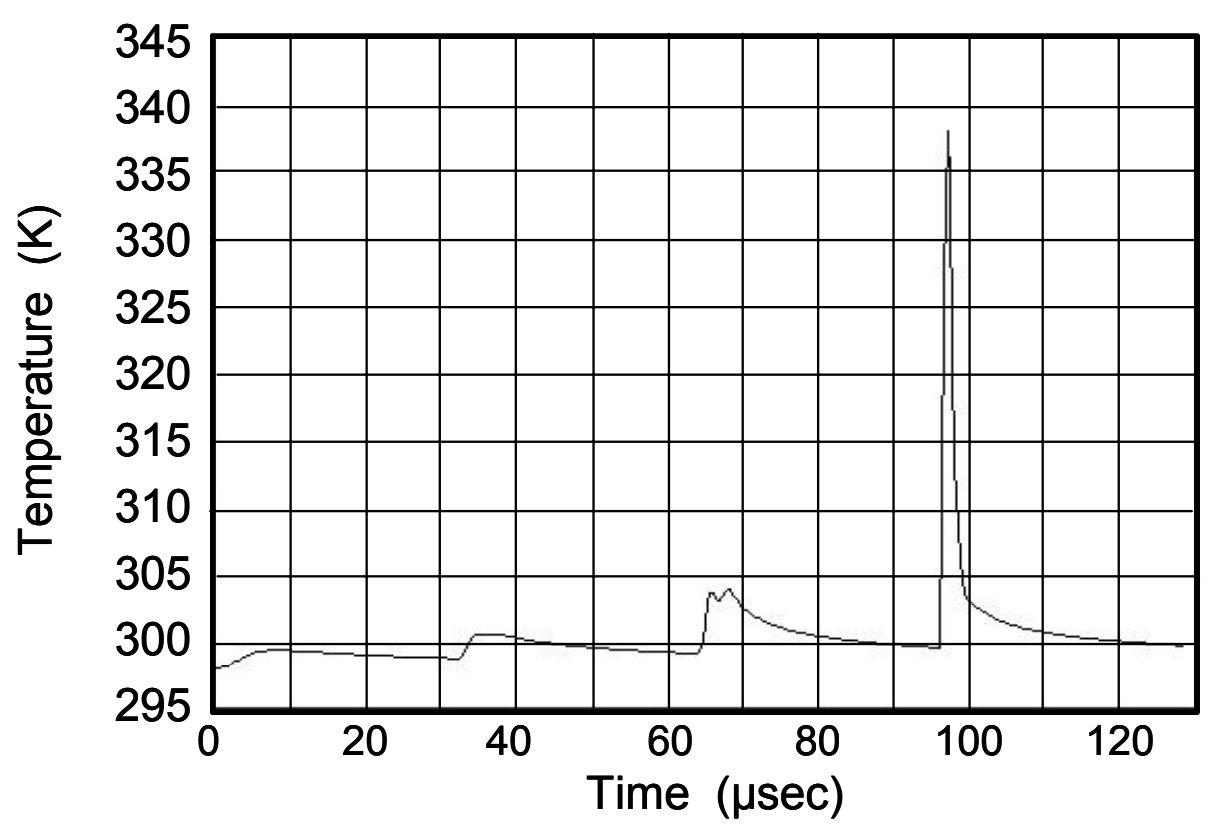

Fig. 5.30 Temperature history corresponding to Point 8 in Fig. 5.14 for unidirectional writing. 
Initially, in Section 3.2.4, it was mentioned that only one-eighth of the entire simulation was capable of being run. After reviewing the visualization and time history plots, definite thermal behavioral patterns are seen to repeat at regular intervals. For example, every time the beam reaches the end of the row, similar contour shapes with similar temperature magnitudes are observed. Because of this repetition from one row to the next, it is unnecessary to simulate the entire writing of the pattern. From these initial rows, the intricacies of each exposure at different points in the row can be found. These localized characteristics can be used in combination with the ability to determine the global heating to predict temperatures at any point in the pattern, whether it was directly simulated or not. 


\section{Chapter 6}

\section{Summary and Future work}

\subsection{Summary}

The primary goal of this research is to demonstrate the ability of finite element modeling to predict the thermal response in an optical reticle due to e-beam patterning. It is well known that the temperature history of the reticle can cause a change in resist sensitivity, which in turn may lead to critical dimension errors in the mask fabrication process, and is therefore important to industry.

Throughout this work, examples are presented to show the capabilities of the ANSYS program to simulate e-beam patterning. Figures included in the thesis present various aspects of the ANSYS program, demonstrating that ANSYS is a suitable choice for analyzing thermal effects due to e-beam patterning. These aspects include the ability to visualize the thermal contours within the material layers, as seen, for example, in Fig. 4.4, and the ability to predict contours at any time during the patterning, an example of which can be seen in Fig. 5.1. An additionally useful feature stems from the fact that the temperature history at any node in the model can be extracted. Knowledge of the temperature history at every point is required to determine the temperature effect on the resist sensitivity, which is the primary concern. None of these capabilities are easily obtainable by experimental methods because of the small field of focus and the extremely fast flash times. 
Two models were created to test the ANSYS simulation program. Model I is a simple test case simulation consisting of three donut shaped flashes. Although the results are informative, the flash configurations are not of industrial interest. Model II, a checkerboard pattern using $2 \mu \mathrm{m} \times 2 \mu \mathrm{m}$ squares, is a more industrially applicable case. Two writing schemes were simulated, unidirectional and serpentine.

From these simulations, two major conclusions were reached. The first is that due to the regularity of the checkerboard pattern, the thermal patterns from row to row are also similar. Therefore, it is only necessary to simulate the first few rows of the pattern per subfield. The only difference from row to row is a slight overall temperature rise in the reticle. This general heating of the reticle is called global heating and can be simulated using finite element methods independent of the localized modeling. The global heating results can be superimposed on the localized heating results, and the thermal response due to patterning of the remaining subfield can be estimated from the results of the first few rows.

This ability to estimate the thermal response is significant because of the large computational resources that would be needed to simulate the entire subfield. For example, if in Model II, the complete subfield were to be patterned, it would take roughly 448 days to complete, and require $1024 \mathrm{~Gb}$ of storage space, assuming the model is run on an 8 processor SGI Origin 2000 machine. The current model currently requires only 7 days to complete and takes up $16 \mathrm{~Gb}$ of storage. The 
patterns required in industry are also fairly regular throughout the subfield so the global heating estimation method described above can be used.

The second conclusion was found when comparing the results of the serpentine and unidirectional writing schemes. The results show that the peak temperatures observed during patterning are higher for the serpentine writing than they are for the unidirectional writing. These higher temperatures occur when the beam turns a corner to begin the next row of patterning. In the region that the beam makes this U-turn, there are a high number of flashes in one area, and as a result the heat diffusion from one flash has more of an effect on the subsequent flashes than in the unidirectional write case, where the beam skips to the beginning of the next line before resuming patterning. It can be deduced that if errors due to localized heating were to occur they would most likely happen during serpentine writing near the areas where the beam turns.

\subsection{Future Work}

A simulation using Model II takes approximately seven days on an eight processor SGI Origin 2000. In order to more effectively use resources, the model must be streamlined to run faster without sacrificing accuracy. Some initial studies to reduce the number of elements have improved the simulation time by a factor of two. However, the results from the faster model and the original model were not exactly the same. More work must be done to investigate the causes of these differences. 
This thesis is only concerned with the thermal portion of the e-beam patterning problem. Ultimately, the goal is to predict areas where unexpected resist development may occur so the patterning can be redesigned. To accomplish this goal requires that the results from the thermal analysis found in this thesis be combined with additional data and calculations. A proposed procedure and description of the additional information required follows:

Using the methods presented in this thesis, the temperature history of each point in the model is calculated. The resist sensitivity history at each point due to its temperature history is then determined using experimental data, which will be in the form of plots of resist sensitivity versus temperature. Next, the electron impact history of each point in the model is calculated. The electron impacts, due to direct exposure or scattering, at each point are found by using results from monte carlo simulations. An example of a monte carlo simulation is found in Fig. 2.4. Finally, the percent of resist development at each point is calculated. This final calculation is done by taking the sensitivity history of each point and combining it with the electron impact history. 


\section{References}

${ }^{1}$ Entry for dope (2001). Webopedia. http://webopedia.internet.com/TERM/d/dope.html (March 14, 2001)

${ }^{2}$ Wylie, Andrew. The early history of the transistor. The Transistor Collector. http://ourworld.compuserve.com/homepages/Andrew_Wylie/history.htm (March 14, 2001)

${ }^{3}$ How chips are made (2001). Intel Corp. http://www.intel.com/education/chips/shock.htm (March 14, 2001)

${ }^{4}$ How transistors work (2001). Intel Corp. http://intel.com/education/transworks/shock.htm (March 14, 2001)

${ }^{5}$ Inventors of the modern computer (2001). About.com. http://www.inventors.about.com/science/inventors/library/weekly/aa080498.htm (March 14, 2001)

${ }^{6}$ Hennesy, John L. (2001). Fundamentals of computer design. http://hennessycube.stanford.edu/chap01-2001.pdf (March 14, 2001)

${ }^{7}$ Basic photolithography process. Semiconductor Consulting Services, Inc. http://www.semiconsulting.com/dl/10370a.pdf (March 14, 2001)

${ }^{8}$ S. Babin, "Measurement of Resist Heating in Photomask Fabrication," J. Vac. Sci. Technol. B, Vol. 15, No. 6, pp. 2209-2213, 1997.

${ }^{9}$ H. Sakuai, T. Abe, M. Itoh, A. Kumagae, H. Anze, and I. Higashikawa, "Resist Heating Effect on $50 \mathrm{keV}$ EB Mask Writing," BACUS News, Vol. 15, No. 8, pp. $1-8,1988$.

${ }^{10}$ K. Harada and S. Sugawara, "Temperature Effects on Positive Electron Resists Irradiated with Electron Beam and Deep-UV Light,” J. Appl. Polym. Sci., Vol. 27, pp. 1441-1452, 1982.

${ }^{11}$ E. Kratschmer and T.R. Groves, "Resist Heating Effects in 25 and $50 \mathrm{kV}$ E-Beam Lithography on Glass Masks,” J. Vac. Sci. Technol. B, Vol. 8, No. 6, pp. 18981902, 1990. 
12 T.R. Groves, "Theory of Beam-Induced Substrate Heating," J. Vac. Sci. Technol. B, Vol. 14, No. 6, pp. 3839-3844, 1996.

${ }^{13}$ E. van der Drift, A. C. Enters, and S. Radelaar, "Thermal Effects in High Voltage E-Beam Lithography,” J. Vac. Sci. Technol. B, Vol. 9, No. 6, pp. 3470-3474, 1991.

${ }^{14}$ N. K. Eib and R. J. Kvitek, "Thermal Distribution and the Effect on Resist Sensitivity in Electron-Beam Direct Write," J. Vac. Sci. Technol. B, Vol. 7, No. 6, pp. 1502-1506, 1989.

${ }^{15}$ T. Abe, K. Ohta, H. Wada, and T. Takigawa, "Resist Heating Effect in Direct Electron Beam Writing,” J. Vac. Sci. Technol. B, Vol. 6, No. 3, pp. 853-857, 1988.

${ }^{16}$ L. Vicum, "Local Heating During Electron Beam Patterning of Lithography Masks," Master's thesis, University of Wisconsin-Madison, Madison, WI 1998.

${ }^{17}$ S. Babin, H. Hartmann, and I. Kuzmin, "Simulation and Measurement of Resist Heating in Multipass Exposure Using a 50 kV Variably Shaped Beam System," Microelectron Eng., Vol. 46, pp. 231-234, 1999.

${ }^{18}$ Bacus 99. S. V. Babin, I. Kostic, and P. Hudek, "Experimental Investigation of the Resist Heating Effect in a Variably Shaped EBL System," Microelectron. Eng., Vol. 21, pp. 173-176, 1993.

${ }^{19}$ S. Babin, "Resist Heating with Different Writing Strategies for High-Throughput Maskmaking,” Microelectron. Eng., Vol. 53, pp. 341-344, 1999.

${ }^{20}$ S. Babin, P. Hudek, and I. Kostic, "Quantitative Measurement of the Resist Heating in a Variable Shaped Electron Lithography," J. Vac. Sci. Technol. B, Vol. 15, No. 2, pp. 311-315, 1997.

${ }^{21}$ TEMPTATION is a registered trademark of Soft Services, P.O. Box 2083, Castro Valley, CA 94546.

${ }^{22}$ S. Babin and I. Y. Kuzmin, "Experimental Verification of the TEMPTATION (Temperature Simulation) Software Tool," J. Vac. Sci. Technol. B, Vol. 16, No. 6, pp. 3241-3247, 1998.

${ }^{23}$ ANSYS is a registered trademark of ANSYS, Inc., Southpointe, 275 Technology Drive, Canonsburg, PA 15317. 
${ }^{24}$ ABAQUS is a registered trademark of Hibbitt, Karlsson \& Sorensen, Inc., 1080 Main Street, Pawtucket, RI 02860. 